\title{
Time Optimal Control of System Governed by a Fractional Stochastic Partial Differential Inclusion with Clarke Subdifferential
}

\author{
Zuomao Yan
}

\begin{abstract}
This paper investigates the time optimal control problems to a new class of fractional non-instantaneous impulsive stochastic partial differential inclusions with Clarke subdifferential in Hilbert spaces. Firstly, using the fractional calculus, properties of fractional resolvent operators and a fixed-point theorem, the existence of mild solutions for these systems is presented. Secondly, the existence of time optimal control of system governed by fractional stochastic control inclusions with Clarke subdifferential and non-instantaneous impulses is also obtained. Finally, an example is given to illustrate our main results.
\end{abstract}

\section{Introduction}

The optimal control theory has a considerable development, and one can find applications in many domains (see $911,13,33]$ ). The time optimal control is an important topic in the theory of optimal controls and plays a vital role in control systems. In the past decades, much attention has been paid to time optimal control problems governed by nonlinear differential systems $14,22,38$. Among them, the fractional time optimal control of a distributed system is an optimal control problem for which dynamic systems are defined by means of fractional differential equations and inclusions, see for example $24,27,29$ and references therein. In particular, it is well known that differential equations with impulsive conditions were used to describe the evolution process with abrupt perturbation at some certain time in biology, medicine and control theory 25. However, in many cases, the models with abrupt and instantaneous impulses can not characterize many practical problems (see $2,2,19,20,26,28,31)$ ). Recently, Wang et al. 37] established the time optimal control of a system governed by a class of non-instantaneous impulsive differential equations in Banach spaces.

On the other hand, stochastic optimal control theory is a stochastic generalization of classical optimal control theory. There are many interesting results on the time optimal Received February 25, 2020; Accepted August 23, 2020.

Communicated by Haomin Zhou.

2010 Mathematics Subject Classification. 34A37, 60H15, 34K50, 26A33, 93E20.

Key words and phrases. time optimal control, fractional non-instantaneous impulsive stochastic partial differential inclusions, fractional resolvent operators, Clarke subdifferential, fixed point.

This work is supported by the National Natural Science Foundation of China (11461019). 
control of stochastic systems [5, 35]. Furthermore, impulsive stochastic optimal control systems describing these optimal control dynamical systems subject to both impulse and stochastic changes have attracted considerable attention [1, 4, 39, 42]. From a practical point of view, many physical phenomena in evolution processes are modeled as impulsive stochastic differential systems for which the impulses are non-instantaneous. Therefore, the optimal control of such systems have become an important object of investigation stimulated by their numerous applications to problems arising in many branches of physics and technical science. For example, Yan and $\mathrm{Lu}$ [40] considered the optimal control of a class of first-order non-instantaneous impulsive stochastic neutral evolution integrodifferential equations with infinite delay. The authors in [41] established the solvability and optimal control of a class of fractional non-instantaneous impulsive stochastic partial integro-differential equations with delay-dependent states. In this paper, we study the time optimal control of non-instantaneous impulsive fractional stochastic partial differential inclusions with Clarke subdifferential of the form

$$
\begin{gathered}
{ }^{c} D_{t}^{\alpha} \mathcal{N}\left(x_{t}\right) \in A \mathcal{N}\left(x_{t}\right)+J_{t}^{1-\alpha}[B(t) u(t)]+J_{t}^{1-\alpha}\left[\mu\left(\partial F\left(t, x_{t}\right)\right) \frac{d w(t)}{d t}\right], \\
t \in\left(s_{i}, t_{i+1}\right], i=0,1, \ldots, N, \\
x(t) \in g_{i}\left(t, x_{t}\right), \quad t \in\left(t_{i}, s_{i}\right], i=1, \ldots, N, \\
x(t)=\varphi(t), \quad t \in(-\infty, 0],
\end{gathered}
$$

where the state $x(\cdot)$ takes values in a separable real Hilbert space $H$ with the inner product $\langle\cdot, \cdot\rangle_{H}$ and the norm $\|\cdot\|_{H},{ }^{c} D_{t}^{\alpha}$ is the Caputo fractional derivative of order $\alpha \in(0,1), J_{t}^{1-\alpha}$ is the $(1-\alpha)$-order fractional integral operator. $A: D(A) \subset H \rightarrow H$ is the infinitesimal generator of resolvent operator $T_{\alpha}(t)(t \geq 0)$. The control function $u$ takes value from a separable Hilbert space $Y$, and $B$ is a linear operator from $Y$ into $H, p \geq 2$ is an integer. Let $K$ be another separable Hilbert space with the inner product $\langle\cdot, \cdot\rangle_{K}$ and the norm $\|\cdot\|_{K} \cdot L(K, H)$ is the space of bounded linear operators mapping $K$ into $H$ equipped with the usual norm $\|\cdot\|_{H}$, and let $L(H)$ denote the Hilbert space of bounded linear operators from $H$ to $H$. Suppose that $\{w(t): t \geq 0\}$ is a given $K$-valued Wiener process with a covariance operator $Q \geq 0$ defined on a complete probability space $(\Omega, \mathcal{F}, P)$ equipped with a normal filtration $\left\{\mathcal{F}_{t}\right\}_{t \geq 0}$, which is generated by the Wiener process $w$. The time history $x_{t}:(-\infty, 0] \rightarrow H$ given by $x_{t}(\theta)=x(t+\theta)$ belongs to some abstract phase space $\mathcal{B}$ defined axiomatically; let $0=t_{0}=s_{0}<t_{1} \leq s_{1}<t_{2} \leq s_{2}<\cdots<t_{N} \leq s_{N}<t_{N+1}=b$ be prefixed numbers. $g_{i}:[0, b] \times \mathcal{B} \rightarrow \mathcal{P}(H)(i=1,2, \ldots, N)$ are bounded, closed, convex-valued multivalued maps, and $G:[0, b] \times \mathcal{B} \rightarrow H$ is appropriate function, $\mathcal{N}(\psi)=\psi(0)-G(t, \psi), \psi \in \mathcal{B}$, $\mu: H \rightarrow L(K, H)$ is a linear compact operator, $\partial F(t, \cdot)$ is the Clarke's subdifferential of $F(t, \cdot)$. The initial data $\{\varphi(t):-\infty<t \leq 0\}$ is an $\mathcal{F}_{0}$-adapted, $\mathcal{B}$-valued random variable independent of the Wiener process $w$ with finite $p$ th moment. 
At present, most of the literatures about differential inclusion require that the nonlinear term is a convex function, which limits the study of differential inclusion. Just as said in [16, it is important to consider, in many problems, functionals on nonconvex constraints. Problems of this kind are typical of variational inequalities, optimization and optimal control theory. The study of the differential inclusions with Clarke subdifferential, which has wider applications than the subdifferential of convex function. In fact, the Clarke subdifferential has important applications in mechanics and engineering, especially in nonsmooth analysis and optimization (see [6,7]). Therefore, it is necessary and important to study differential inclusions with Clarke subdifferential type. Very recently, the papers 18,23 studied the optimal control results for fractional evolution inclusion and fractional impulsive evolution inclusion with Clarke subdifferential in Banach spaces. To the best of our knowledge no work has been reported in the present literature regarding the time optimal control for fractional impulsive stochastic partial differential inclusions in Hilbert spaces, which is expressed in the form (1.1)-(1.3). Motivated by the above consideration, we study this interesting problem, which is natural generalization of time optimal control concept well known in the theory of infinite dimensional control systems. In the present paper, we investigate the time optimal control of (1.1)-(1.3) under the mixed Lipschitz and Carathéodory conditions. The results are obtained by using theory of fractional resolvent operators, stochastic analysis, the properties of Clarke subdifferential with a fixed-point theorem. The known results appeared in $14,18,22,24,27,29,37,38$ are generalized to the impulsive stochastic inclusions settings and the case of with infinite delay and multi-valued non-instantaneous impulses.

The rest of this paper is organized as follows. In Section 2 , we introduce some notations and necessary preliminaries. In Section 3, we give the existence of solutions for control system. In Section 4, we establish the existence of time optimal control. In Section 5, an example is given to illustrate our results.

\section{Preliminaries}

We assume that there exists a complete orthonormal system $\left\{e_{n}\right\}_{n=1}^{\infty}$ in $K$, a bounded sequence of nonnegative real numbers $\left\{\lambda_{n}\right\}_{n=1}^{\infty}$ such that $Q e_{n}=\lambda_{n} e_{n}$, and a sequence $\alpha_{n}$ of independent Brownian motions such that

$$
\langle w(t), e\rangle_{K}=\sum_{n=1}^{\infty} \sqrt{\lambda_{n}}\left\langle e_{n}, e\right\rangle_{K} \alpha_{n}(t), \quad e \in K, t \geq 0 .
$$

Let $L_{2}^{0}=L_{2}\left(Q^{1 / 2} K, H\right)$ be the space of all Hilbert-Schmidt operators from $Q^{1 / 2} K$ to $H$ with the norm $\|\psi\|_{L_{2}^{0}}^{2}=\operatorname{Tr}\left(\left(\psi Q^{1 / 2}\right)\left(\psi Q^{1 / 2}\right)^{*}\right)$ for any $\psi \in L_{2}^{0}$. Clearly for any bounded operators $\psi \in L(K, H)$ this norm reduces to $\|\psi\|_{L_{2}^{0}}^{2}=\operatorname{Tr}\left(\psi Q \psi^{*}\right)$. Let $L_{\mathcal{F}_{t}}^{p}(\Omega, H)$ be the 
Banach space of all $\mathcal{F}_{t}$-measurable $p$ th power integrable random variables with values in the Hilbert space $H$. Let $C\left([0, b] ; L^{p}(\Omega, H)\right)$ be the Banach space of continuous maps from $[0, b]$ into $L^{p}(\Omega, H)$ satisfying the condition $\sup _{t \in[0, b]} E\|x(t)\|_{H}^{p}<\infty$.

We use the notations $\mathcal{P}(H)=\left\{x \in 2^{H}: x \neq \emptyset\right\}$. Let $V$ and $Z$ be two Hausdorff topological spaces. A multi-valued map $\Phi: V \rightarrow 2^{Z} \backslash\{\emptyset\}$ is said to be upper semicontinuous (for short u.s.c.) $x_{0} \in V$, if for any neighborhood $O\left(\Phi\left(x_{0}\right)\right)$ of $\Phi\left(x_{0}\right)$, there exists a neighborhood $O\left(x_{0}\right)$ of $x_{0}$ such that $\Phi(x) \subset O\left(\Phi x_{0}\right)$ for all $x \in O\left(x_{0}\right)$; compact if $\Phi(D)$ is relatively compact for every any bounded subset $D$ of $V$; quasicompact if its restriction to any compact subset $D \subset V$ is compact. Consider $H_{d}: \mathcal{P}(H) \times$ $\mathcal{P}(H) \rightarrow \mathbb{R}^{+} \cup\{\infty\}$ given by $H_{d}(\widetilde{A}, \widetilde{B})=\max \left\{\sup _{\widetilde{a} \in \widetilde{A}} d(\widetilde{a}, \widetilde{B}), \sup _{\widetilde{b} \in \widetilde{B}} d(\widetilde{A}, \widetilde{a})\right\}$, where $d(\widetilde{A}, \widetilde{b})=\inf _{\widetilde{a} \in \widetilde{A}} d(\widetilde{a}, \widetilde{b}), d(\widetilde{a}, \widetilde{B})=\inf _{\widetilde{b} \in \widetilde{B}} d(\widetilde{a}, \widetilde{b})$. Then, $\left(\mathcal{P}_{\mathrm{bd}, \mathrm{cl}}(H), H_{d}\right)$ is a metric space and $\left(\mathcal{P}_{\mathrm{cl}}(H), H_{d}\right)$ is a generalized metric space. For more details, one can see [12].

We introduce the space $\mathcal{P C}(H)$ formed by all $\mathcal{F}_{t}$-adapted measurable, $H$-valued stochastic processes $\{x(t): t \in[0, b]\}$ such that $x$ is continuous at $t \neq t_{i}, x\left(t_{i}\right)=x\left(t_{i}^{-}\right)$and $x\left(t_{i}^{+}\right)$ exists for all $i=1, \ldots, N$. In this paper, we always assume that $\mathcal{P C}(H)$ is endowed with the norm $\|x\|_{\mathcal{P C}}=\left(\sup _{0 \leq t \leq b} E\|x(t)\|_{H}^{p}\right)^{1 / p}$. Then $\left(\mathcal{P C}(H),\|\cdot\|_{\mathcal{P C}}\right)$ is a Banach space. The notation $B_{r}(x, H)$ stands for the closed ball with center at $x$ and radius $r>0$ in $H$.

In this paper, we assume that the phase space $\left(\mathcal{B},\|\cdot\|_{\mathcal{B}}\right)$ is a semi-normed linear space of $\mathcal{F}_{0}$-measurable functions mapping $(-\infty, 0]$ into $H$, and satisfying the following fundamental axioms due to Hale and Kato (see, e.g., [17]):

(A) If $x:(-\infty, \sigma+b] \rightarrow H, b>0$, is such that $\left.x\right|_{[\sigma, \sigma+b]} \in C([\sigma, \sigma+b], H)$ and $x_{\sigma} \in \mathcal{B}$, then for every $t \in[\sigma, \sigma+b]$ the following conditions hold:

(i) $x_{t}$ is in $\mathcal{B}$;

(ii) $\|x(t)\|_{H} \leq \widetilde{H}\left\|x_{t}\right\|_{\mathcal{B}}$;

(iii) $\left\|x_{t}\right\|_{\mathcal{B}} \leq K(t-\sigma) \sup \left\{\|x(s)\|_{H}: \sigma \leq s \leq t\right\}+M(t-\sigma)\left\|x_{\sigma}\right\|_{\mathcal{B}}$, where $\widetilde{H} \geq 0$ is a constant; $K, M:[0, \infty) \rightarrow[1, \infty), K$ is continuous and $M$ is locally bounded, and $\widetilde{H}, K, M$ are independent of $x(\cdot)$.

(B) For the function $x(\cdot)$ in $(\mathrm{A}), x_{t}$ is a $\mathcal{B}$-valued function on $[\sigma, \sigma+b]$.

(C) The space $\mathcal{B}$ is complete.

In the following, let $Y$ be a separable Hilbert space from which the controls $u$ take the values. Operator $B \in L_{\infty}([0, b], L(Y, H)),\|B\|_{\infty}$ stands for the norm of operator $B$ on Banach space $L_{\infty}([0, b], L(Y, H))$, where $L_{\infty}([0, b], L(Y, H))$ denotes the space of operator valued functions which are measurable in the strong operator topology and uniformly bounded on the interval $[0, b]$. Let $L_{\mathcal{F}}^{p}([0, b], Y)$ be the closed subspace of $L_{\mathcal{F}}^{p}([0, b] \times$ 
$\Omega, Y)$, consisting of all measurable and $\mathcal{F}_{t}$-adapted, $Y$-valued stochastic processes satisfying the condition $E \int_{0}^{b}\|u(t)\|_{Y}^{p} d t<\infty$, and endowed with the norm $\|u\|_{L_{\mathcal{F}}^{p}([0, b], Y)}=$ $\left(E \int_{0}^{b}\|u(t)\|_{Y}^{p} d t\right)^{1 / p}$.

Let $U$ be a nonempty closed bounded convex subset of $Y$. We define the admissible control set

$$
U_{\text {ad }}=\left\{\varpi(\cdot) \in L_{\mathcal{F}}^{p}([0, b], Y): \varpi(t) \in U \text { a.e. } t \in[0, b]\right\} .
$$

Then, $B u \in L^{p}([0, b], H)$ for all $u \in U_{\text {ad }}$.

We recall some basic definitions about fractional integral and derivative, we refer to 34 .

Definition 2.1. [7] Let $X$ be a Banach space with the dual space $X^{*}$ and $F: X \rightarrow \mathbb{R}$ be a locally Lipschitz functional on $X$. The Clarke's generalized directional derivative of $F$ at the point $x \in X$ in the direction $v \in X$, denoted by $F^{0}(x, v)$, is defined by

$$
F^{0}(x, v)=\lim _{\lambda \rightarrow 0^{+}} \sup _{y \rightarrow x} \frac{F(y+\lambda v)-F(y)}{\lambda} .
$$

The Clarke's generalized gradient of $F$ at $x \in X$, denoted by $\partial F(x)$, is a subset of $X^{*}$ given by

$$
\partial F(x)=\left\{x^{*} \in X^{*}: F^{0}(x, v) \geq\left\langle x^{*}, v\right\rangle, \forall v \in X\right\} .
$$

Definition 2.2. 36 A family $\left\{T_{\alpha}(t)\right\}_{t \geq 0} \subseteq L(H)$ of bounded linear operators in $H$ is called an $\alpha$-order fractional resolvent operator (or $\alpha$-order fractional solution operator) if it satisfies the following conditions:

(i) $T_{\alpha}(t)$ is strongly continuous for $t \geq 0$ and $T_{\alpha}(0)=I$.

(ii) $T_{\alpha}(t) D(A) \subset D(A)$ and $A T_{\alpha}(t) x=T_{\alpha}(t) A x$ for all $x \in D(A), t \geq 0$.

(iii) The resolvent equation holds for all $x \in D(A), t \geq 0$,

$$
T_{\alpha}(t) x=x+\int_{0}^{t} g_{\alpha}(t-s) A T_{\alpha}(s) x d s,
$$

where $g_{\alpha}(t-s)=\frac{(t-s)^{\alpha}}{\Gamma(\alpha)}$.

Definition 2.3. 36 Let $0<\theta_{0}<\pi / 2$ and $\omega_{0} \in \mathbb{R}$. An $\alpha$-order fractional resolvent operator $T_{\alpha}(t)(t \geq 0)$ is called analytic if it admits an analytic extension to a sector $\Sigma_{\theta_{0}}:=\left\{z \in \mathbb{C} \backslash\{0\}:|\arg z|<\theta_{0}\right\}$ and the analytic extension is strongly continuous on $\Sigma_{\theta}$ for every $\theta \in\left(0, \theta_{0}\right)$. An analytic $\alpha$-order fractional resolvent operator $T_{\alpha}(z)\left(z \in \Sigma_{\theta}\right)$ is said to be of analyticity type $\left(\omega_{0}, \theta_{0}\right)$ if for each $\theta \in\left(0, \theta_{0}\right)$ and $\omega>\omega_{0}$, there exists a positive constant $M=M(\omega, \theta)$ such that

$$
\left\|T_{\alpha}(z)\right\|_{H} \leq M e^{\omega \operatorname{Re} z}, \quad z \in \Sigma_{\theta} .
$$


An analytic $\alpha$-order fractional resolvent operator $T_{\alpha}(z)\left(z \in \Sigma_{\theta}\right)$ is said to be bounded if for each $\theta \in\left(0, \theta_{0}\right)$, there exists a positive constant $M_{\theta}$ such that

$$
\left\|T_{\alpha}(z)\right\|_{H} \leq M_{\theta}, \quad z \in \Sigma_{\theta}
$$

Let us consider the linear fractional stochastic differential inclusions of the form

$$
\begin{gathered}
{ }^{c} D_{t}^{\alpha} x(t) \in A x(t)+J_{t}^{1-\alpha}[B(t) u(t)]+J_{t}^{1-\alpha}\left[\mu(\partial F(t)) \frac{d w(t)}{d t}\right], \quad t \in[0, b], \\
x(0)=\varphi(0) \in \mathcal{B},
\end{gathered}
$$

where function $\partial F \in L^{p}([0, b], H)$.

If $x$ satisfies the inclusions 2.1$]-2.2$, then for $t \in[0, b]$, we have

$$
x(t)=\varphi(0)+A\left(g_{\alpha} * x\right)(t)+\int_{0}^{t} B(s) u(s) d s+\int_{0}^{t} \mu f(s) d w(s),
$$

where $f \in L^{p}([0, b], H), f(t) \in \partial F(t)$ a.e. $t \in[0, b]$. Using the definition of an $\alpha$-resolvent and the definition of an integral solution, it follows that

$$
\begin{aligned}
I * x & =\left(T_{\alpha}-A g_{\alpha} * T_{\alpha}\right) * x=T_{\alpha} *\left[x-\left(A g_{\alpha} * x\right)\right] \\
& =T_{\alpha} *[x-(x-\varphi(0)-I * B u-I * \mu f)]=T_{\alpha} *[\varphi(0)+I * B u+I * \mu f] \\
& =T_{\alpha} * \varphi(0)+I * T_{\alpha} * B u+I * T_{\alpha} * \mu f,
\end{aligned}
$$

where $*$ is the convolution. Then we have for all $t \in[0, b]$

$$
x(t)=T_{\alpha}(t) \varphi(0)+\int_{0}^{t} T_{\alpha}(t-s) B(s) u(s) d s+\int_{0}^{t} T_{\alpha}(t-s) \mu f(s) d w(s), \quad t \in[0, b]
$$

is the variation of constant formula satisfying (2.1)-(2.2). Now, we define the mild solution for $2.1-2.2$ as follows:

Definition 2.4. A function $x \in C([0, b], H)$ is called a mild solution of $(2.1)-(2.2)$ if $x_{0}=\varphi \in \mathcal{B}$ on $(-\infty, 0]$, and $(2.3)$ holds for every $u \in U_{\text {ad }}$, where $f \in L^{p}([0, b], H)$, $f(t) \in \partial F(t)$ a.e. $t \in[0, b]$.

Let $x:(-\infty, b] \rightarrow H$ be a function such that $x \in \mathcal{P C}(H)$. If $x$ is a solution of (1.1)(1.3), from Definition 2.4 and the partial neutral differential inclusions theory, we obtain

$$
\begin{aligned}
x(t)= & T_{\alpha}(t)[\varphi(0)-G(0, \varphi)]+G\left(t, x_{t}\right)+\int_{0}^{t} T_{\alpha}(t-s) B(s) u(s) d s \\
& +\int_{0}^{t} T_{\alpha}(t-s) \mu f(s) d w(s), \quad t \in\left[0, t_{1}\right],
\end{aligned}
$$


where $f \in L^{p}([0, b], H), f(t) \in \partial F\left(t, x_{t}\right)$ a.e. $t \in[0, b]$. If $t=s_{1}$, then $x\left(s_{1}\right)=\widetilde{g}_{1}\left(s_{1}\right)$, where $\widetilde{g}_{1}\left(s_{1}\right) \in g_{1}\left(s_{1}, x_{s_{1}}\right)$. For $t \in\left(s_{1}, t_{2}\right]$, we have

$$
\begin{aligned}
x(t)= & T_{\alpha}\left(t-s_{1}\right)\left[x\left(s_{1}\right)-G\left(s_{1}, x_{s_{1}}\right)\right]+G\left(t, x_{t}\right)+\int_{s_{1}}^{t} T_{\alpha}(t-s) B(s) u(s) d s \\
& +\int_{s_{1}}^{t} T_{\alpha}(t-s) \mu f(s) d w(s) \\
= & T_{\alpha}\left(t-s_{1}\right)\left[\widetilde{g}_{1}\left(s_{1}\right)-G\left(s_{1}, x_{s_{1}}\right)\right]+G\left(t, x_{t}\right)+\int_{s_{1}}^{t} T_{\alpha}(t-s) B(s) u(s) d s \\
& +\int_{s_{i}}^{t} T_{\alpha}(t-s) \mu f(s) d w(s) .
\end{aligned}
$$

By repeating the same procedure, we can easily deduce that

$$
\begin{aligned}
x(t)= & T_{\alpha}\left(t-s_{i}\right)\left[\widetilde{g}_{i}\left(s_{i}\right)-G\left(s_{i}, x_{s_{i}}\right)\right]+G\left(t, x_{t}\right)+\int_{s_{i}}^{t} T_{\alpha}(t-s) B(s) u(s) d s \\
& +\int_{s_{i}}^{t} T_{\alpha}(t-s) \mu f(s) d w(s)
\end{aligned}
$$

holds for all $t \in\left(s_{i}, t_{i+1}\right]$, where $\widetilde{g}_{i}(t) \in g_{i}\left(t, x_{t}\right), i=1, \ldots, N$.

Next we will derive the appropriate definition of mild solutions of $1.1-1.3$.

Definition 2.5. An $\mathcal{F}_{t}$-adapted stochastic process $x:(-\infty, b] \rightarrow H$ is called a mild solution of the system $[1.1-1.3)$ if $x_{0}=\varphi \in \mathcal{B}$ on $(-\infty, 0],\left.x\right|_{[0, b]} \in \mathcal{P C}([0, b], H)$, and

(i) $x(t)$ is measurable and adapted to $\mathcal{F}_{t}, t \geq 0$.

(ii) $x(t) \in H$ has càdlàg paths on $t \in[0, b]$ a.s and for each $t \in[0, b], x(t)$ satisfies $x(t)=\widetilde{g}_{j}(t)$ for all $t \in\left(t_{j}, s_{j}\right], j=1, \ldots, N$, and

$$
\begin{aligned}
x(t)= & T_{\alpha}(t)[\varphi(0)-G(0, \varphi)]+G\left(t, x_{t}\right)+\int_{0}^{t} T_{\alpha}(t-s) B(s) u(s) d s \\
& +\int_{0}^{t} T_{\alpha}(t-s) \mu f(s) d w(s)
\end{aligned}
$$

for all $t \in\left[0, t_{1}\right]$, and

$$
\begin{aligned}
x(t)= & S_{\alpha}\left(t-s_{i}\right)\left[\widetilde{g}_{i}\left(s_{i}\right)-G\left(s_{i}, x_{s_{i}}\right)\right]+G\left(t, x_{t}\right)+\int_{s_{i}}^{t} T_{\alpha}(t-s) B(s) u(s) d s \\
& +\int_{s_{i}}^{t} T_{\alpha}(t-s) \mu f(s) d w(s)
\end{aligned}
$$

for all $t \in\left(s_{i}, t_{i+1}\right], i=1, \ldots, N$, where $u \in U_{\mathrm{ad}}, f \in L^{p}([0, b], H), f(t) \in \partial F\left(t, x_{t}\right)$ a.e. $t \in[0, b]$, and $\widetilde{g}_{i}(t) \in g_{i}\left(t, x_{t}\right), i=1, \ldots, N$. 
Definition 2.6. 15 A multi-valued map $\Phi: H \rightarrow 2^{H}$ is referred to as condensing if, for any bounded subset $D \subseteq H$, which is not relatively compact, one has $\beta(\Phi(D))<\beta(D)$, where $\beta(\cdot)$ stands for the Kuratowski measure of noncompactness.

Lemma 2.7. 15 Let $X$ and $V$ be two Hausdorff topological spaces. Assume that $\Phi: X \rightarrow$ $2^{V} \backslash\{\emptyset\}$ is a multi-valuedmap such that $\Phi(X) \subset \widetilde{K}$ and the graph of $\Phi$ is closed, where $\widetilde{K}$ is a compact set. Then $\Phi$ is u.s.c.

The next result is a consequence of the phase space axioms.

Lemma 2.8. Let $x:(-\infty, b] \rightarrow H$ be an $\mathcal{F}_{t}$-adapted measurable process such that the $\mathcal{F}_{0}$-adapted process $x_{0}=\varphi(t) \in L_{\mathcal{F}_{0}}^{p}(\Omega, \mathcal{B})$ and $\left.x\right|_{[0, b]} \in \mathcal{P C}([0, b], H)$, then

$$
\left\|x_{s}\right\|_{\mathcal{B}} \leq M_{b} E\|\varphi\|_{\mathcal{B}}+K_{b} \sup _{0 \leq s \leq b} E\|x(s)\|_{H},
$$

where $K_{b}=\sup \{K(t): 0 \leq t \leq b\}, M_{b}=\sup \{M(t): 0 \leq t \leq b\}$.

Lemma 2.9. [8] For any $p \geq 1$ and for arbitrary $L_{2}^{0}(K, H)$-valued predictable process $\phi(\cdot)$ such that

$$
\sup _{s \in[0, t]} E\left\|\int_{0}^{s} \phi(v) d w(v)\right\|_{H}^{2 p} \leq(p(2 p-1))^{p}\left(\int_{0}^{t}\left(E\|\phi(s)\|_{L_{2}^{0}}^{2 p}\right)^{1 / p} d s\right)^{p}, \quad t \in[0, \infty) .
$$

Lemma 2.10. [12] Let $D$ be a nonempty, bounded, closed subset of a Banach space $H$, and $\Phi: D \rightarrow \mathcal{P}(D)$ be a u.s.c. condensing multi-valued map. If for every $x \in D, \Phi(x)$ is a closed and convex subset of $D$, then $\Phi$ admits a fixed point in $D$.

\section{Existence of mild solutions}

In this section, we prove the existence of mild solutions for the system $(1.1)-(1.3)$. Assume that the following hypotheses:

(H1) $T_{\alpha}(t)$ is an analytic resolvent of analyticity type $\left(\omega_{0}, \theta_{0}\right)$ and $M=\sup _{t \in[0, b]}\left\|T_{\alpha}(t)\right\|_{H}$ $<\infty$.

(H2) The function $G:[0, b] \times \mathcal{B} \rightarrow H$ is continuous, and there exists a positive constant $L_{G}$ such that

$$
\begin{array}{clrl}
E\left\|G\left(t_{1}, \psi_{1}\right)-G\left(t_{2}, \psi_{2}\right)\right\|_{H}^{p} \leq L_{G}\left[\left|t_{1}-t_{2}\right|+\left\|\psi_{1}-\psi_{2}\right\|_{\mathcal{B}}^{p}\right], & & t \in[0, b], \psi_{1}, \psi_{2} \in \mathcal{B}, \\
E\|G(t, \psi)\|_{H}^{p} \leq L_{G}\left(\|\psi\|_{\mathcal{B}}^{p}+1\right), & & t \in[0, b], \psi \in \mathcal{B} .
\end{array}
$$

(H3) The functional $F:[0, b] \times \mathcal{B} \rightarrow \mathbb{R}$ satisfies the following conditions:

(i) $F(t, \cdot):[0, b] \rightarrow \mathbb{R}$ is measurable for all $\psi \in \mathcal{B}$. 
(ii) $F(t, \psi): \mathcal{B} \rightarrow \mathbb{R}$ is locally Lipschitz continuous for a.e. $t \in[0, b]$.

(iii) There exist functions $\left\{\Lambda_{n^{*}}\right\} \subset L^{1}\left([0, b], \mathbb{R}^{+}\right)$such that

$$
\sup _{\|\psi\|_{\mathcal{B}}^{p} \leq n^{*}} E\|\partial F(t, \psi)\|_{H}^{p} \leq \Lambda_{n^{*}}(t)
$$

for a.e. $t \in[0, b], n^{*}>0$ with

$$
\liminf _{n^{*} \rightarrow+\infty} \frac{\left\|\Lambda_{n^{*}}\right\|_{L^{1}}}{n^{*}}=0
$$

where $E\|\partial F(t, \psi)\|_{H}^{p}=\sup \left\{E\|f\|_{H}^{p}: f \in \partial F(t, \psi)\right\}$.

(H4) There exist $\gamma_{i}>0, i=1, \ldots, N$, such that

$$
\begin{array}{rlrl}
E H_{d}^{p}\left(g_{i}\left(t_{1}, \psi_{1}\right), g_{i}\left(t_{2}, \psi_{2}\right)\right) & \leq \gamma_{i}\left[\left|t_{1}-t_{2}\right|+\left\|\psi_{1}-\psi_{2}\right\|_{\mathcal{B}}^{p}\right], & & t \in\left(t_{i}, s_{i}\right], \psi_{1}, \psi_{2} \in \mathcal{B}, \\
E H_{d}^{p}\left(g_{i}(t, \psi), 0\right) \leq \gamma_{i}\left(\|\psi\|_{\mathcal{B}}^{p}+1\right), & & t \in\left(t_{i}, s_{i}\right], \psi \in \mathcal{B} .
\end{array}
$$

Let $q$ satisfy $1 / p+1 / q=1$ and an operator $\Psi: L^{q}([0, b], H) \rightarrow \mathcal{P}\left(L^{p}([0, b], H)\right)$ be defined by

$$
\Psi(\psi)=\left\{f \in L^{p}([0, b], H): f(t) \in \partial F(t, \psi) \text { a.e. } t \in[0, b]\right\}, \quad \psi \in L^{q}([0, b], H) .
$$

We also need some auxiliary results.

Lemma 3.1. (Compare with [30]) Let condition (H3) hold and the operator $\Psi$ satisfy: if $x_{n} \rightarrow x \in L^{q}([0, b], H), f_{n} \stackrel{w}{\longrightarrow} f \in L^{p}([0, b], H)$ and $f_{n} \in \Psi\left(x_{n}\right)$. Then one has $f \in \Psi(x)$.

Lemma 3.2. (Compare with $[23]$ ) Let condition (H3) hold. Then for every $x \in L^{q}([0, b], H)$, the set $\Psi(x)$ is nonempty weakly compact convex.

Now, we are in a position to state the existence theorem

Theorem 3.3. If assumptions (H1)-(H4) are satisfied, then for each $u \in U_{\mathrm{ad}}$, the system (1.1) - 1.3 has at least one mild solution on $[0, b]$, provided that

$$
2^{p-1} K_{b}^{p} \max _{1 \leq i \leq N}\left[\left(8^{p-1} M^{p}+1\right) \gamma_{i}+4^{p-1}\left(2^{p-1} M^{p}+1\right) L_{G}\right]<1 .
$$

Proof. Consider the space $\mathcal{Y}=\left\{x:(-\infty, b] \rightarrow H ; x(0)=\varphi(0),\left.x\right|_{[0, b]} \in \mathcal{P C}(H)\right\}$ endowed with the uniform convergence topology and consider the operator $\Phi: \mathcal{Y} \rightarrow \mathcal{P}(\mathcal{Y})$ defined by

$$
\Phi(x)=\{v \in \mathcal{Y}: v(t) \text { satifies } 3.2\}
$$


where 3.2 is given by

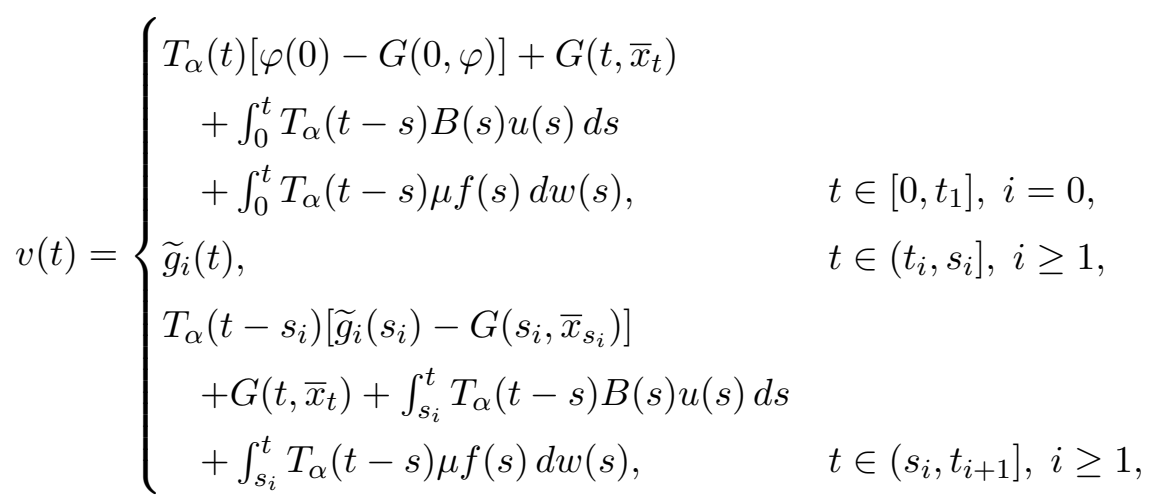

and $\bar{x}:(-\infty, 0] \rightarrow H$ is such that $\bar{x}_{0}=\varphi$ and $\bar{x}=x$ on $[0, b]$, where $f(t) \in \Psi\left(\bar{x}_{t}\right)=\{f \in$ $L^{p}([0, b], H): f(t) \in \partial F\left(t, \bar{x}_{t}\right)$ a.e. $\left.t \in[0, b]\right\}, \bar{x}_{t} \in \mathcal{B}$, and $\widetilde{g}_{i}(t) \in g_{i}\left(t, \bar{x}_{t}\right), i=1, \ldots, N$. From (H1) and Hölder's inequality, the following inequality holds:

$$
\begin{aligned}
E\left\|\int_{s_{i}}^{t} T_{\alpha}(t-s) B(s) u(s) d s\right\|_{H}^{p} & \leq E\left[\int_{s_{i}}^{t}\left\|T_{\alpha}(t-s)\right\|_{H}\|B(s) u(s)\|_{H} d s\right]^{p} \\
& \leq M^{p}\|B\|_{\infty}^{p}\left(t_{i+1}-s_{i}\right)^{p-1} E \int_{s_{i}}^{t}\|u(s)\|_{Y}^{p} d s \\
& \leq M^{p}\|B\|_{\infty}^{p}\left(t_{i+1}-s_{i}\right)^{p-1}\|u\|_{L_{\mathcal{F}}^{p}([0, b], Y)}^{p} .
\end{aligned}
$$

Then from Bochner's Theorem, it follows that $T_{\alpha}(t-s) B(s) u(s)$ are integrable on $\left(s_{i}, t\right)$, $i=0,1, \ldots, N$. It is clear that $\Phi$ is a well-defined operator on $\mathcal{Y}$. We shall show that $\Phi$ satisfies the hypotheses of Lemma 2.10. The proof will be given in several steps.

Step 1: There exists $n \in \mathbb{N}$ such that $\Phi\left(B_{n}\right) \subset B_{n}$, where $B_{n}=\left\{x \in \mathcal{Y}:\|x\|_{\mathcal{P C}}^{p} \leq n\right\}$. To the contrary, assume that there exist $\left\{x_{n}\right\},\left\{v_{n}\right\} \subset \mathcal{Y}$ such that $x_{n} \in B_{n}, v_{n} \in \Phi\left(x_{n}\right)$ and $v_{n} \notin B_{n}, n \in \mathbb{N}$. Then, there exist sequences $\left\{f_{n}\right\}$ and $\left\{\widetilde{g}_{i, n}\right\}$ with $f_{n}(t) \in \Psi\left(\bar{x}_{n, t}\right)$ and $\widetilde{g}_{i, n}(t) \in g_{i}\left(t, \bar{x}_{n, t}\right)$, such that

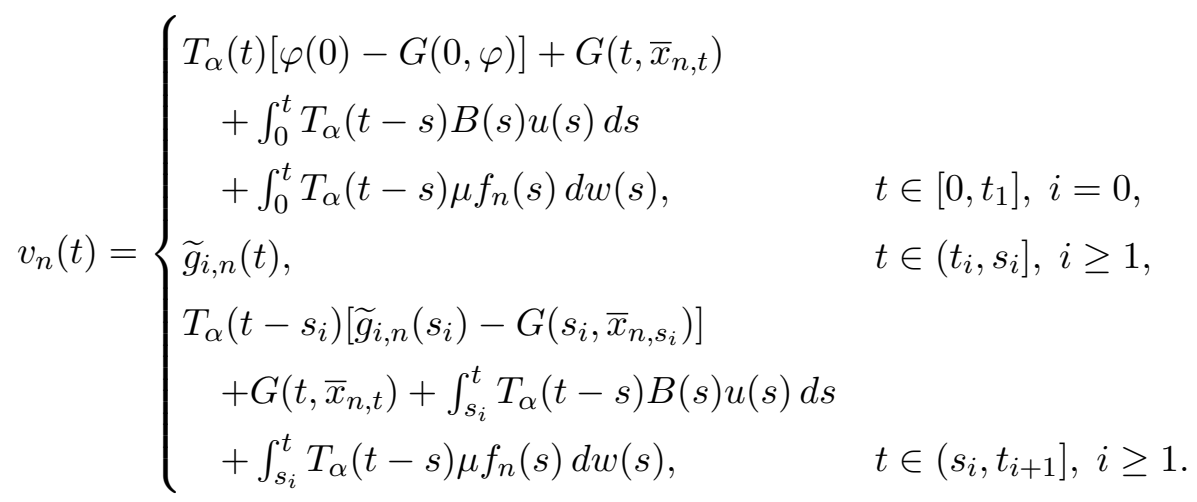

If $x \in B_{n}$, from Lemma 2.8 , it follows that

$$
\left\|\bar{x}_{s}\right\|_{\mathcal{B}}^{p} \leq 2^{p-1}\left[M_{b}\|\varphi\|_{\mathcal{B}}\right]^{p}+2^{p-1} K_{b}^{p} n:=n^{*} .
$$


By (H1)-(H3), we have for $t \in\left[0, t_{1}\right]$,

$$
\begin{aligned}
E\left\|v_{n}(t)\right\|_{H}^{p} \leq & 4^{p-1} E\left\|T_{\alpha}(t)[\varphi(0)-G(0, \varphi)]\right\|_{H}^{p}+4^{p-1} E\left\|G\left(t, \bar{x}_{n, t}\right)\right\|_{H}^{p} \\
& +4^{p-1} E\left\|\int_{0}^{t} T_{\alpha}(t-s) B(s) u(s) d s\right\|_{H}^{p} \\
& +4^{p-1} E\left\|\int_{0}^{t} T_{\alpha}(t-s) \mu f_{n}(s) d w(s)\right\|_{H}^{p} \\
\leq & 8^{p-1} M^{p}\left[E\|\varphi(0)\|_{H}^{p}+L_{G}\left(\|\varphi\|_{\mathcal{B}}^{p}+1\right)\right]+4^{p-1} L_{G}\left(\left\|\bar{x}_{t}\right\|_{\mathcal{B}}^{p}+1\right) \\
& +4^{p-1} M^{p}\|B\|_{\infty}^{p} t_{1}^{p-1}\|u\|_{L_{\mathcal{F}}^{p}\left(\left[0, t_{1}\right], Y\right)}^{p} \\
& +4^{p-1} C_{p} M^{p}\|\mu\|_{L(H)}^{p}\left[\int_{0}^{t}\left[E\left\|f_{n}(s)\right\|_{L_{2}^{0}}^{p}\right]^{2 / p} d s\right]^{p / 2} \\
\leq & 8^{p-1} M^{p}\left[E\|\varphi(0)\|_{H}^{p}+L_{G}\left(\|\varphi\|_{\mathcal{B}}^{p}+1\right)\right]+4^{p-1} L_{G}\left(n^{*}+1\right) \\
& +4^{p-1} M^{p}\|B\|_{\infty}^{p} t_{1}^{p-1}\|u\|_{L_{\mathcal{F}}^{p}\left(\left[0, t_{1}\right], Y\right)}^{p} \\
& +4^{p-1} C_{p} M^{p}\|\mu\|_{L(K, H)}^{p} t_{1}^{p / 2-1}\left\|\Lambda_{n^{*}}\right\|_{L^{1}\left(\left[0, t_{1}\right], \mathbb{R}^{+}\right)} .
\end{aligned}
$$

For any $t \in\left(t_{i}, s_{i}\right], i=1, \ldots, N$; by (H4), we have

$$
E\left\|v_{n}(t)\right\|_{H}^{p} \leq \gamma_{i}\left(\left\|\bar{x}_{t}\right\|_{\mathcal{B}}^{p}+1\right) \leq \gamma_{i}\left(n^{*}+1\right) .
$$

Similarly, for any $t \in\left(s_{i}, t_{i+1}\right], i=1, \ldots, N$, we have

$$
\begin{aligned}
E\left\|v_{n}(t)\right\|_{H}^{p} \leq & 4^{p-1} E\left\|T_{\alpha}\left(t-s_{i}\right)\left[\widetilde{g}_{i, n}\left(s_{i}\right)-G\left(s_{i}, \bar{x}_{n, s_{i}}\right)\right]\right\|_{H}^{p} \\
& +E\left\|G\left(t, \bar{x}_{n, t}\right)\right\|_{H}^{p}+E\left\|\int_{s_{i}}^{t} T_{\alpha}(t-s) B(s) u(s) d s\right\|_{H}^{p} \\
& +E\left\|\int_{s_{i}}^{t} T_{\alpha}(t-s) \mu f_{n}(s) d w(s)\right\|_{H}^{p} \\
\leq & 8^{p-1} M^{p}\left[\gamma_{i}\left(n^{*}+1\right)+L_{G}\left(n^{*}+1\right)\right]+4^{p-1} L_{G}\left(n^{*}+1\right) \\
& +4^{p-1} M^{p}\|\mu\|_{L(H)}^{p}\|B\|_{\infty}^{p}\left(t_{i+1}-s_{i}\right)^{p-1}\|u\|_{L_{\mathcal{F}}^{p}\left(\left[s_{i}, t_{i+1}\right], Y\right)}^{p} \\
& +4^{p-1} C_{p} M^{p}\|\mu\|_{L(K, H)}^{p}\left(t_{i+1}-s_{i}\right)^{p / 2-1}\left\|\Lambda_{n^{*}}\right\|_{L^{1}\left(\left[s_{i}, t_{i+1}\right], \mathbb{R}^{+}\right)} .
\end{aligned}
$$

Then, for all $t \in[0, b]$, we have

$$
\begin{aligned}
n<E\left\|v_{n}(t)\right\|_{H}^{p} \leq & M_{*}+\max _{1 \leq i \leq N}\left\{\left(8^{p-1} M^{p}+1\right) \gamma_{i}+4^{p-1}\left(2^{p-1} M^{p}+1\right) L_{G}\right\} n^{*} \\
& +4^{p-1} C_{p} M^{p}\|\mu\|_{L(K, H)}^{p} b^{p / 2-1}\left\|\Lambda_{n^{*}}\right\|_{L^{1}\left([0, b], \mathbb{R}^{+}\right)}
\end{aligned}
$$

where

$$
\begin{aligned}
M_{*}= & 8^{p-1} M^{p}\left[E\|\varphi(0)\|_{H}^{p}+L_{G}\left(\|\varphi\|_{\mathcal{B}}^{p}+1\right)\right] \\
& +\max _{1 \leq i \leq N}\left\{\left(8^{p-1} M^{p}+1\right) \gamma_{i}+4^{p-1}\left(2^{p-1} M^{p}+1\right) L_{G}\right\} \\
& +4^{p-1} M^{p}\|B\|_{\infty}^{p} b^{p-1}\|u\|_{L_{\mathcal{F}}^{p}([0, b], Y)}^{p} .
\end{aligned}
$$


Thus,

$$
\begin{aligned}
n^{*}<2^{p-1}\left[M_{b}\|\varphi\|_{\mathcal{B}}\right]^{p}+2^{p-1} K_{b}^{p}\left[M_{*}+\max _{1 \leq i \leq N}\left\{\left(8^{p-1} M^{p}+1\right) \gamma_{i}+4^{p-1}\left(2^{p-1} M^{p}+1\right) L_{G}\right\} n^{*}\right. \\
\left.+4^{p-1} C_{p} M^{p}\|\mu\|_{L(K, H)}^{p} b^{p / 2-1}\left\|\Lambda_{n^{*}}\right\|_{L^{1}\left([0, b], \mathbb{R}^{+}\right)}\right]
\end{aligned}
$$

Dividing on both sides by $n^{*}$ and taking the lower limit as $n^{*} \rightarrow \infty$, we get

$$
2^{p-1} K_{b}^{p} \max _{1 \leq i \leq N}\left\{\left(8^{p-1} M^{p}+1\right) \gamma_{i}+4^{p-1}\left(2^{p-1} M^{p}+1\right) L_{G}\right\} \geq 1 .
$$

This is a contradiction with the formula (3.1). Hence for some $n \in \mathbb{N}, \Phi\left(B_{n}\right) \subset B_{n}$.

Step 2: $\Phi(x)$ is convex for each $x \in \mathcal{Y}$. In fact, if $v^{1}, v^{2}$ belong to $\Phi(x)$, then there exist $f_{1}(t), f_{2}(t) \in \Psi\left(\bar{x}_{t}\right)$ and $\widetilde{g}_{i, 1}(t), \widetilde{g}_{i, 2}(t) \in g_{i}\left(t, \bar{x}_{t}\right)$ for $t \in\left[0, t_{1}\right]$ such that

$$
\begin{aligned}
v^{k}(t)= & T_{\alpha}(t)[\varphi(0)-G(0, \varphi)]+G\left(t, \bar{x}_{t}\right) \\
& +\int_{0}^{t} T_{\alpha}(t-s) B(s) u(s) d s+\int_{0}^{t} T_{\alpha}(t-s) \mu f_{k}(s) d w(s), \quad k=1,2 .
\end{aligned}
$$

Let $0 \leq \lambda \leq 1$. For $t \in\left[0, t_{1}\right]$ we have

$$
\begin{aligned}
\left(\lambda v^{1}+(1-\lambda) v^{2}\right)(t)= & T_{\alpha}(t)[\varphi(0)-G(0, \varphi)]+G\left(t, \bar{x}_{t}\right)+\int_{0}^{t} T_{\alpha}(t-s) B(s) u(s) d s \\
& +\int_{0}^{t} T_{\alpha}(t-s) \mu\left[\lambda f_{1}(s)+(1-\lambda) f_{2}(s)\right] d w(s) .
\end{aligned}
$$

For any $t \in\left(t_{i}, s_{i}\right], i=1, \ldots, N$, we have

$$
\left(\lambda v^{1}+(1-\lambda) v^{2}\right)(t)=\left[\lambda \widetilde{g}_{i, 1}(t)+(1-\lambda) \widetilde{g}_{i, 2}(t)\right]
$$

Similarly, for any $t \in\left(s_{i}, t_{i+1}\right], i=1, \ldots, N$, we have

$$
\begin{aligned}
v^{k}(t)= & T_{\alpha}\left(t-s_{i}\right)\left[\widetilde{g}_{i, k}\left(s_{i}\right)-G\left(s_{i}, \bar{x}_{s_{i}}\right)\right]+G\left(t, \bar{x}_{t}\right) \\
& +\int_{s_{i}}^{t} T_{\alpha}(t-s) B(s) u(s) d s+\int_{s_{i}}^{t} T_{\alpha}(t-s) \mu f_{k}(s) d w(s), \quad k=1,2 .
\end{aligned}
$$

Let $0 \leq \lambda \leq 1$. For each $t \in\left(s_{i}, t_{i+1}\right], i=1, \ldots, N$, we have

$$
\begin{aligned}
\left(\lambda v^{1}+(1-\lambda) v^{2}\right)(t)= & T_{\alpha}\left(t-s_{i}\right)\left\{\left[\lambda \widetilde{g}_{i, 1}\left(s_{i}\right)+(1-\lambda) \widetilde{g}_{i, 2}\left(s_{i}\right)\right]-G\left(s_{i}, \bar{x}_{s_{i}}\right)\right\}+G\left(t, \bar{x}_{t}\right) \\
& +\int_{s_{i}}^{t} T_{\alpha}(t-s) B(s) u(s) d s \\
& +\int_{s_{i}}^{t} T_{\alpha}(t-s) \mu\left[\lambda f_{1}(s)+(1-\lambda) f_{2}(s)\right] d w(s) .
\end{aligned}
$$

In view of Lemma 3.2 and $g_{i}(i=1,2, \ldots, N)$ are convex values, we have $\left(\lambda v^{1}+(1-\lambda) v^{2}\right) \in$ $\Phi(x)$. 
Step 3: $\Phi(x)$ is closed for each $x \in \mathcal{Y}$. Let $\left\{v_{n}\right\}_{n \geq 0} \in \Phi(x)$ such that $v_{n} \rightarrow v$ in $\mathcal{Y}$. Then there exists $f_{n}(t) \in \Psi\left(\bar{x}_{n, t}\right)$ and $\widetilde{g}_{i, n}(t) \in g_{i}\left(t, \bar{x}_{n, t}\right)$ such that

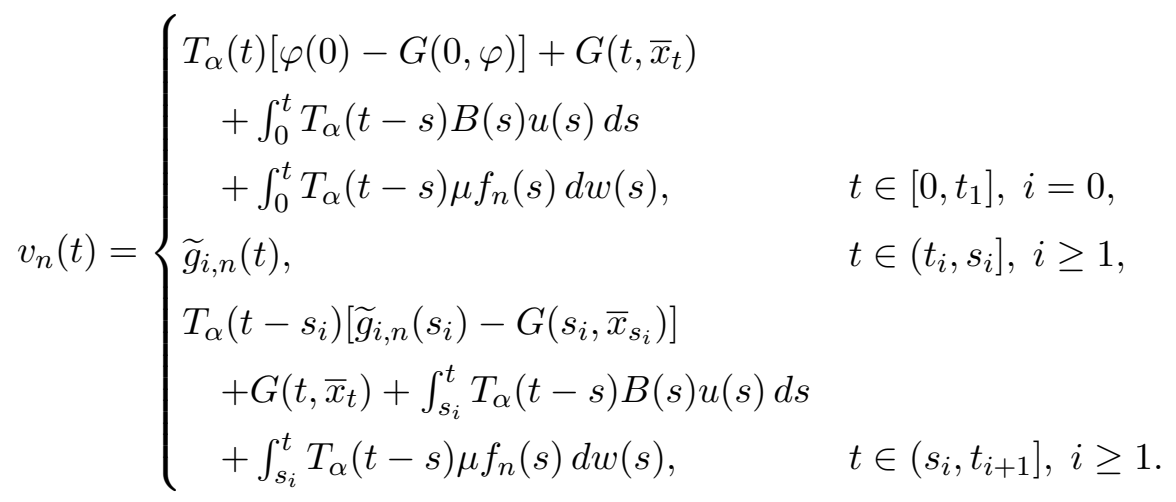

By Lemma 3.2 , passing to a subsequence if necessary, we can deduce that $f_{n} \stackrel{w}{\longrightarrow} f$ in $L^{p}([0, b], H)$. By applying condition (H3), we know that $\left\{f_{n}\right\} \subset L^{p}([0, b], H)$ is bounded. Thus, there exists a subsequence, denoted it again by $\left\{f_{n}\right\}$, which converges weakly to $f^{*}$ in $L^{p}([0, b], H)$. By the uniqueness of the limit, it is easy to have $f=f^{*}$. Since $g_{i}$, $i=1, \ldots, N$, are closed operators, it follows that $\widetilde{g}_{i, n}(t) \rightarrow \widetilde{g}_{i}(t) \in g_{i}\left(t, \bar{x}_{t}\right)$ for every $t \in\left(t_{i}, s_{i}\right], i=1, \ldots, N$. Therefore, we have for each $t \in[0, b]$,

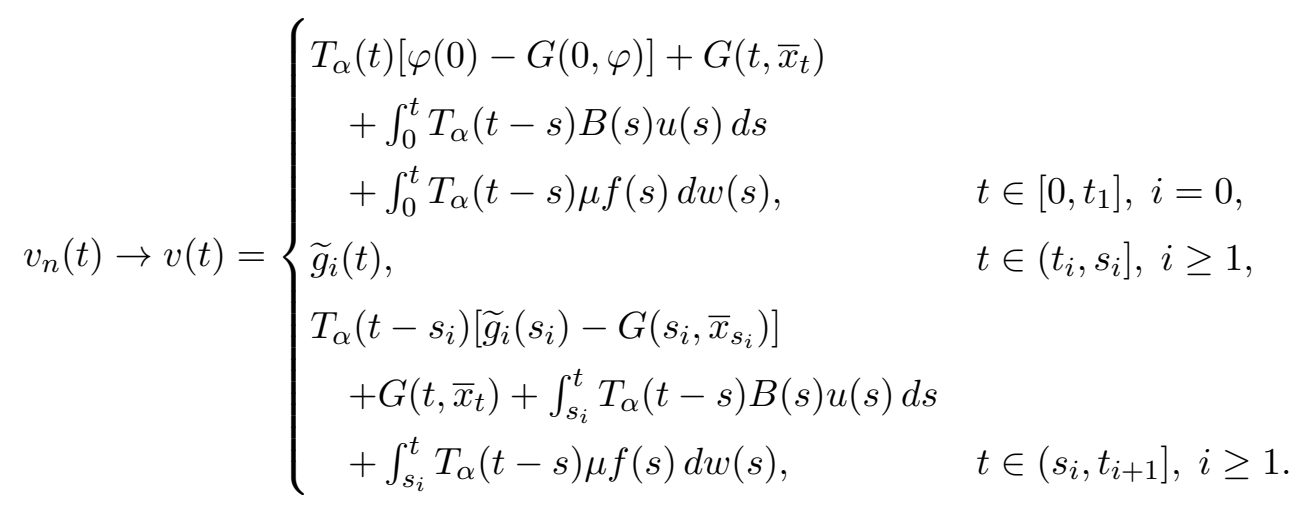

This shows that $v \in \Phi(x)$.

Step 4: $\Phi(x)$ is u.s.c. and condensing. For this purpose, we decompose $\Phi$ as $\Phi=$ $\Phi_{1}+\Phi_{2}$, where the operators $\Phi_{1}, \Phi_{2}$ are defined on $B_{n}$ respectively by

$$
\left(\Phi_{1} x\right)(t)= \begin{cases}T_{\alpha}(t)[\varphi(0)-G(0, \varphi)]+G\left(t, \bar{x}_{t}\right), & t \in\left[0, t_{1}\right], i=0, \\ \widetilde{g}_{i}(t), & t \in\left(t_{i}, s_{i}\right], i \geq 1, \\ T_{\alpha}\left(t-s_{i}\right)\left[\widetilde{g}_{i}\left(s_{i}, \bar{x}_{s_{i}}\right)-G\left(s_{i}, \bar{x}_{s_{i}}\right)\right]+G\left(t, \bar{x}_{t}\right), & t \in\left(s_{i}, t_{i+1}\right], i \geq 1\end{cases}
$$

and

$$
\Phi_{2}(x)=\{\zeta \in \mathcal{Y}: v(t) \text { satifies }(3.3)\},
$$


where 3.3 is given by

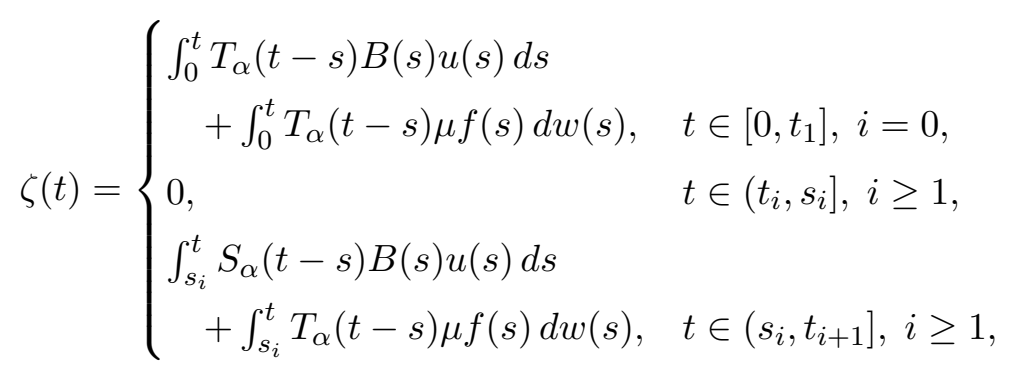

where $f(t) \in \Psi\left(\bar{x}_{t}\right)$ and $\widetilde{g}_{i}(t) \in g_{i}\left(t, \bar{x}_{t}\right)$. By Lemma 2.10, we need only show that $\Phi_{1}$ is contractive and $\Phi_{2}$ is u.s.c. and compact.

Claim 1: $\Phi_{1}$ is a contraction map on $B_{n}$. Let $t \in\left[0, t_{1}\right]$ and $x^{*}, x^{* *} \in \mathcal{B P C}$. From (H2) and Lemma 2.8, we have

$$
\begin{aligned}
& E H_{d}^{p}\left(\left(\Phi_{1} x^{*}\right)(t),\left(\Phi_{1} x^{* *}\right)(t)\right) \\
\leq & L_{G}\left\|\left(\overline{x^{*}}\right)_{t}-\left(\overline{x^{* *}}\right)_{t}\right\|_{\mathcal{B}}^{p} \\
\leq & 2^{p-1} K_{b}^{p} L_{G} \sup \left\{E\left\|\overline{x^{*}}(\tau)-\overline{x^{* *}}(\tau)\right\|_{H}^{p}, 0 \leq \tau \leq t\right\} \\
\leq & 2^{p-1} K_{b}^{p} L_{G} \sup _{s \in[0, b]} E\left\|\overline{x^{*}}(s)-\overline{x^{* *}}(s)\right\|_{H}^{p} \\
= & \left.2^{p-1} K_{b}^{p} L_{G} \sup _{s \in[0, b]} E\left\|x^{*}(s)-x^{* *}(s)\right\|_{H}^{p} \quad \text { (since } \bar{x}=x \text { on }[0, b]\right) \\
= & 2^{p-1} K_{b}^{p} L_{G}\left\|x^{*}-x^{* *}\right\|_{\mathcal{P C}}^{p} .
\end{aligned}
$$

For any $t \in\left(t_{i}, s_{i}\right], i=1, \ldots, N$, we have

$$
E H_{d}^{p}\left(\left(\Phi_{1} x^{*}\right)(t),\left(\Phi_{1} x^{* *}\right)(t)\right) \leq 2^{p-1} K_{b}^{p} \gamma_{i}\left\|x^{*}-x^{* *}\right\|_{\mathcal{P C}}^{p} .
$$

Similarly, for any $t \in\left(t_{i}, t_{i+1}\right], i=1, \ldots, N$, we have

$$
\begin{aligned}
E H_{d}^{p}\left(\left(\Phi_{1} x^{*}\right)(t),\left(\Phi_{1} x^{* *}\right)(t)\right) \leq & 4^{p-1} M^{p}\left[\gamma_{i}\left\|\left(\overline{x^{*}}\right)_{t}-\left(\overline{x^{* *}}\right)_{t}\right\|_{\mathcal{B}}^{p}+L_{G}\left\|\left(\overline{x^{*}}\right)_{s_{i}}-\left(\overline{x^{* *}}\right)_{s_{i}}\right\|_{\mathcal{B}}^{p}\right] \\
& \left.+2^{p-1} L_{G} \|\left(\overline{x^{*}}\right)_{t}\right)-\left(\overline{x^{* *}}\right)_{t} \|_{\mathcal{B}}^{p} \\
\leq & 4^{p-1} K_{b}^{p}\left[2^{p-1} M^{p} \gamma_{i}+\left(1+2^{p-1} M^{p}\right) L_{G}\right]\left\|x^{*}-x^{* *}\right\|_{\mathcal{P C}}^{p} .
\end{aligned}
$$

Thus, for all $t \in[0, b]$, we have

$$
E H_{d}^{p}\left(\left(\Phi_{1} x^{*}\right)(t),\left(\Phi_{1} x^{* *}\right)(t)\right) \leq L_{0}\left\|x^{*}-x^{* *}\right\|_{\mathcal{P C}}^{p}
$$

Taking supremum over $t$,

$$
E H_{d}^{p}\left(\Phi_{1} x^{*}, \Phi_{1} x^{* *}\right) \leq L_{0}\left\|x^{*}-x^{* *}\right\|_{\mathcal{P C}}^{p},
$$

where $L_{0}=2^{p-1} K_{b}^{p} \max _{1 \leq i \leq N}\left[\left(4^{p-1} M^{p}+1\right) \gamma_{i}+2^{p-1}\left(1+M^{p}\right) L_{G}\right]$. By (3.1), we see that $L_{0}<1$. Hence, $\Phi_{1}$ is a contraction. 
Claim 2: $\Phi_{2} x$ is clearly bounded for all $x \in B_{n}$.

Claim 3: $\Phi_{2}$ is equicontinuous on $B_{n}$. Since $T_{\alpha}(\cdot)$ is strongly continuous, the function $s \rightarrow T_{\alpha}(s)$ is continuous in the uniform operator topology on $(0, b]$. Let $s_{i}<\varepsilon<t \leq t_{i+1}$, $i=0,1, \ldots, N$, and $\delta>0$ such that $\left\|T_{\alpha}\left(\tau_{1}\right)-T_{\alpha}\left(\tau_{2}\right)\right\|_{H}^{p}<\varepsilon$ for every $\tau_{1}, \tau_{2} \in\left(s_{i}, t_{i+1}\right]$ with $\left|\tau_{1}-\tau_{2}\right|<\delta$. For each $x \in B_{n}, 0<|\widetilde{\tau}|<\delta, t, t+\widetilde{\tau} \in\left[s_{i}, t_{i+1}\right], i=1, \ldots, N$, and $\zeta \in \Phi_{2} x$, there exists $f \in \Psi\left(\bar{x}_{t}\right)$, we have

$$
\begin{aligned}
& E\|\zeta(t+\widetilde{\tau})-\zeta(t)\|_{H}^{p} \\
& \leq 4^{p-1} E\left\|\int_{s_{i}}^{t}\left[T_{\alpha}(t+\widetilde{\tau}-s)-T_{\alpha}(t-s)\right] B(s) u(s) d s\right\|_{H}^{p} \\
&+4^{p-1} E\left\|\int_{t}^{t+\widetilde{\tau}} T_{\alpha}(t+\widetilde{\tau}-s) B(s) u(s)(s) d s\right\|_{H}^{p} \\
&+4^{p-1} E\left\|\int_{s_{i}}^{t}\left[T_{\alpha}(t+\widetilde{\tau}-s)-T_{\alpha}(t-s)\right] \mu f(s) d w(s)\right\|_{H}^{p} \\
&+4^{p-1} E\left\|\int_{t}^{t+\widetilde{\tau}} T_{\alpha}(t+\widetilde{\tau}-s) \mu f(s) d w(s)\right\|_{H}^{p} \\
& \leq 4^{p-1}\|B\|_{\infty}^{p}\left(t_{i+1}-s_{i}\right)^{p-1} \int_{s_{i}}^{t}\left\|S_{\alpha}(t+\widetilde{\tau}-s)-S_{\alpha}(t-s)\right\|_{H}^{p} E\|u(s)\|_{H}^{p} d s \\
&+4^{p-1}\|B\|_{\infty}^{p} M^{p} \widetilde{\tau}^{p-1} \int_{t}^{t+\widetilde{\tau}} E\|u(s)\|_{H}^{p} d s \\
&+4^{p-1} C_{p}\left[\int_{s_{i}}^{t}\left\|T_{\alpha}(t+\widetilde{\tau}-s)-T_{\alpha}(t-s)\right\|_{H}^{p}\left[E\|f(s)\|_{H}^{p}\right]^{2 / p}\right]^{p / 2} d s \\
&+4^{p-1} C_{p} M^{p}\|\mu\|_{L(K, H)}^{p} \widetilde{\tau}^{p / 2-1} \int_{t}^{t+\widetilde{\tau}} \Lambda_{n^{*}}(s) d s . \\
&+4^{p-1} C_{p} M^{p}\left[\int_{t}^{t+\widetilde{\tau}}\left[E\|f(s)\|_{H}^{p}\right]^{2 / p} d s\right]_{n^{*}}^{p / 2} d s \\
& \leq 4^{p-1} \varepsilon\|B\|_{\infty}^{p}\left(t_{i+1}-s_{i}\right)^{p-1}\|u\|_{L_{\mathcal{F}}^{p}}^{p}\left(\left(s_{i}, t_{i+1}\right], Y\right) d s \\
&+4^{p-1}\|B\|_{\infty}^{p} M^{p} \widetilde{\tau}^{p-1} \int_{t}^{t+\tau} E\|u(s)\|_{H}^{p} d s \\
& L(K, H) \\
&
\end{aligned}
$$

The right-hand side tends to zero as $\widetilde{\tau} \rightarrow 0$, and sufficiently small positive number $\varepsilon$. Hence $\Phi_{2}$ maps $B_{n}$ into an equicontinuous family of functions.

Claim 4: $\left(\Phi_{2} B_{n}\right)(t)$ is relatively compact in $H$ for each $t \in[0, b]$, where $\left(\Phi_{2} B_{n}\right)(t)=$ $\left\{\zeta(t): \zeta \in \Phi_{2} B_{n}\right\}, t \in[0, b]$. If $\tau, \tau^{\prime} \in\left[\tau_{k}, \tau_{k+1}\right]$ for some $k=1,2, \ldots, n-1$, we can select points $s_{i}=\tau_{1}<\tau_{2}<\cdots<\tau_{n}=t$ such that $\left\|T_{\alpha}(t-\tau)-T_{\alpha}\left(t-\tau^{\prime}\right)\right\| \leq \varepsilon$. For $x \in B_{n}$, 
and $\zeta \in \Phi_{2} x$, there exists $f(t) \in \Psi\left(\bar{x}_{t}\right)$, we have

$$
E\left\|\int_{s_{i}}^{t} f(s) d w(s)\right\|_{H}^{p} \leq C_{p}\left(t_{i+1}-s_{i}\right)^{p / 2-1} \int_{s_{i}}^{t_{i+1}} \Lambda_{n}(s) d s=l_{i}^{*} .
$$

Then we find that

$$
\begin{aligned}
\int_{s_{i}}^{t} T_{\alpha}(t-s) \mu f(s) d w(s)= & \sum_{k=1}^{n-1} \int_{\tau_{k}}^{\tau_{k+1}}\left[T_{\alpha}(t-s)-T_{\alpha}\left(t-\tau_{k}\right)\right] \mu f(s) d w(s) \\
& +\sum_{k=1}^{n-1} T_{\alpha}\left(t-\tau_{k}\right) \mu \int_{\tau_{k}}^{\tau_{k+1}} f(s) d w(s) \\
\in & \mathcal{D}_{i, \varepsilon}+\sum_{k=1}^{n-1} T_{\alpha}\left(t-\tau_{k}\right) \mu B_{l_{i}^{*}}(0, H)
\end{aligned}
$$

By the compactness of $\mu$, we have $\left\{\int_{s_{i}}^{t} T_{\alpha}(t-s) \mu f(s) d w(s): x \in B_{n}\right\} \in \mathcal{D}_{i, \varepsilon}+\mathcal{J}_{i, \varepsilon}$, where $\operatorname{diam}\left(\mathcal{D}_{i, \varepsilon}\right) \leq \varepsilon\|\mu\|_{L(K, H)}^{p} l_{i}^{*}$ and $\mathcal{J}_{i, \varepsilon}$ are relatively compact, which permit us concluding that the set $\left\{\int_{s_{i}}^{t} T_{\alpha}(t-s) \mu f(s) d w(s): x \in B_{n}\right\}$ is relatively compact in $H$ since $\varepsilon$ is arbitrary. On the other hand, the set $\left\{\int_{s_{i}}^{t} T_{\alpha}(t-s) B(s) u(s) d s\right\}$ is bounded and independent of $x$. Thus $\left(\Phi_{2} B_{n}\right)(t)$ is relatively compact in $H$ for each $t \in[0, b]$.

Step 5: $\Phi_{2}$ has a closed graph. Let $x^{(n)} \rightarrow x^{*}(n \rightarrow \infty), \zeta^{(n)} \in \Phi_{2} x^{(n)}, x^{(n)} \in B_{n}$ and $\zeta^{(n)} \rightarrow \zeta^{*}$. From Axiom $(\mathrm{A})$, it is easy to see that $\left(\overline{x^{(n)}}\right)_{s} \rightarrow\left(\overline{x^{*}}\right)_{s}$ uniformly for $s \in(-\infty, b]$ as $n \rightarrow \infty$. We shall prove that $\zeta^{*} \in \Phi_{2} \overline{x^{*}}$. Now $\zeta^{(n)} \in \Phi \overline{x^{(n)}}$ means that there exists $f^{(n)}(t) \in \Psi\left(\left(\overline{x^{(n)}}\right)_{t}\right)$ such that, for each $t \in\left[s_{i}, t_{i+1}\right], i=1, \ldots, N$,

$$
\zeta^{(n)}(t)=\int_{s_{i}}^{t} T_{\alpha}(t-s) B(s) u(s) d s+\int_{s_{i}}^{t} T_{\alpha}(t-s) \mu f^{(n)}(s) d w(s) .
$$

According to (H3), we obtain that the boundedness of $f^{n}$. In view of the reflexivity of $L^{p}([0, b], H)$, without loss of generality, we can suppose that $f^{n} \stackrel{w}{\longrightarrow} f^{*} \in L^{p}([0, b], H)$. A continuous linear operator $\Upsilon: L^{p}([0, b], H) \rightarrow P C([0, b], H)$ is defined as

$$
\Upsilon(f)(\cdot)=\int_{s_{i}} T_{\alpha}(\cdot-s) B(s) u(s) d s+\int_{s_{i}} T_{\alpha}(\cdot-s) \mu f^{*}(s) d w(s) .
$$

By the compactness of $\mu$, we have

$$
\begin{aligned}
\left\|\Upsilon\left(f^{n}\right)(t)-\Upsilon\left(f^{*}\right)(t)\right\|_{H}^{p} & \leq C_{p} M^{p}\left[\int_{s_{i}}^{t}\left[E\left\|\mu\left[f^{n}(s)-f^{*}(s)\right]\right\|_{H}^{p}\right]^{2 / p} d s\right]^{p / 2} \\
& \leq C_{p} M^{p}\left(t_{i+1}-s_{i}\right)^{p / 2-1} \int_{s_{i}}^{t}\left\|\mu f^{m}(s)-\mu f^{*}(s)\right\|_{H}^{p} d s \\
& \leq C_{p} M^{p}\left(t_{i+1}-s_{i}\right)^{p / 2}\left\|\mu f^{m}-\mu f^{*}\right\|_{\mathcal{P C}}^{p} \quad \text { as } n \rightarrow \infty .
\end{aligned}
$$


Because $x^{(n)} \rightarrow x$ in $\mathcal{Y} \subset L^{q}([0, b], H)$, from Lemma 3.1, it follows that

$$
\zeta^{*}(t)=\int_{s_{i}}^{t} T_{\alpha}(t-s) B(s) u(s) d s+\int_{s_{i}}^{t} T_{\alpha}(t-s) \mu f^{*}(s) d w(s)
$$

for $f^{*}(t) \in \Psi\left(\left(\overline{x^{*}}\right)_{t}\right)$. Therefore, $\Phi_{2}$ has a closed graph and $\Phi_{2}$ is a completely continuous multi-valued map with compact value. So $\Phi_{2}$ is u.s.c. Hence $\Phi=\Phi_{1}+\Phi_{2}$ is u.s.c. and condensing. By Lemma 2.10, $\Phi$ admits a fixed point on $B_{n}$. Therefore, the system (1.1)(1.3) has at least one mild solution $x$ in $B_{n}$. The proof is complete.

\section{Existence of time optimal controls}

In this section, we consider a control problem and present a result on the existence of fractional impulsive stochastic time optimal controls.

Let $x^{u}$ denote the mild solution of system (1.1) 1.3 corresponding to the control $u \in U_{\text {ad }}$. Let $x_{0}, x_{1} \in H$ with $x_{0} \neq x_{1}$. For some $t>0$, we suppose that there exists an admissible control $u$ satisfying $x(t, f, u)=x_{1}$ and $x_{0}=x(0)=\varphi(0)$. Let us define the transition time, which is the first time $t^{u}$ such that $x\left(t^{u}, f^{u}, u\right)=x_{1}$. The optimal time is defined by low limit $t^{0}$ of $t^{u}$ such that $x\left(t^{u}, f^{u}, u\right)=x_{1}$ for admissible control $u$. If the control $u^{0} \in U_{\text {ad }}$ such that $x\left(t^{0}, f^{0}, u^{0}\right)=x_{1}$, then $u^{0} \in U_{\text {ad }}$ is called the time optimal control. It is sufficient to prove that the existence of the admissible control satisfies $x\left(t^{0}, f^{0}, u^{0}\right)=x_{1}$ with respect to $\left(x_{0}, x_{1}\right)$.

Theorem 4.1. Assume that all the hypotheses of Theorem 3.3 hold. Suppose that B is a strongly continuous operator. Then there exists a time optimal control with respect to $\left(x_{0}, x_{1}\right)$.

Proof. Let

$$
t^{0}=\inf \left\{t: x(t, f, u)=x_{1} \text {, where } u \text { is an admissible control }\right\}
$$

Then, there exists a monotone decreasing sequence $t^{n} \rightarrow t^{0}$ as $n \rightarrow \infty$. Assume that $u \in U_{\text {ad }}$ is the corresponding control such that the trajectories

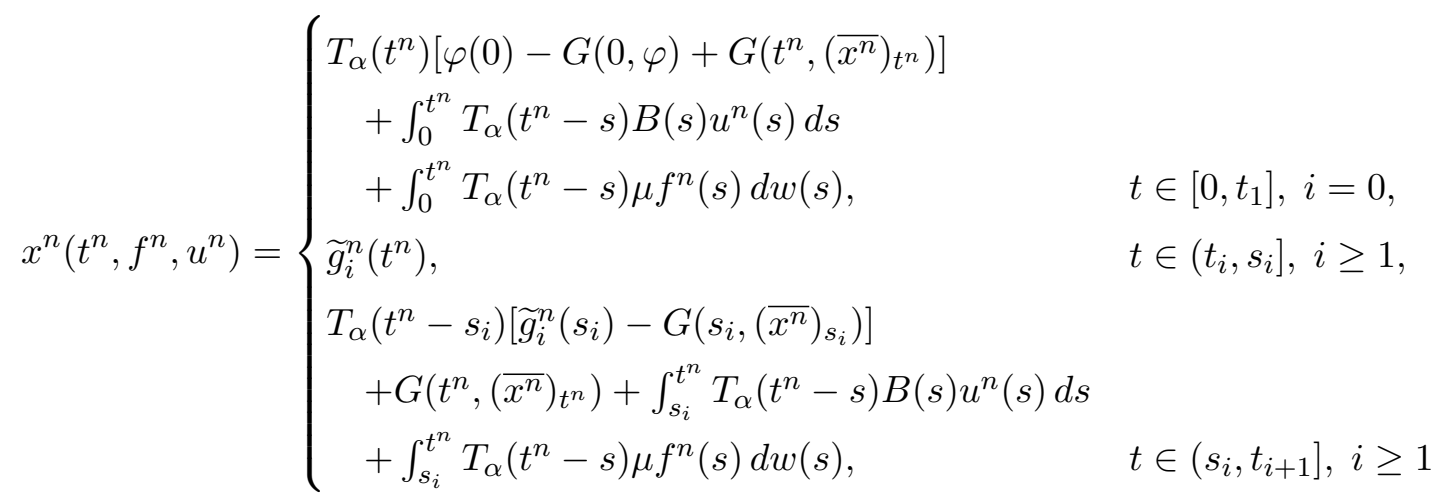


satisfying $x^{n}\left(t^{n}, f^{n}, u^{n}\right)=x_{1}, n=1,2, \ldots$, and $f^{n}(s) \in \Psi\left(\left(\overline{x^{n}}\right)_{s}\right), \widetilde{g}_{i}^{n}\left(t^{n}\right) \in g_{i}\left(t^{n},\left(\overline{x^{n}}\right)_{t^{n}}\right)$. Notice that $x^{n}\left(t^{n}, f^{n}, u^{n}\right) \in B_{n}$. Because $\left\{u^{n}\right\} \subseteq U_{\text {ad }},\left\{u^{n}\right\}$ is bounded in $L_{\mathcal{F}}^{p}([0, b], Y)$, so there exists a subsequence, relabeled as $\left\{u^{n}\right\}$, and $u^{0} \in L_{\mathcal{F}}^{p}([0, b], Y)$ such that

$$
u^{n} \stackrel{w}{\longrightarrow} u^{0} \quad \text { in } L_{\mathcal{F}}^{p}([0, b], Y) \quad \text { as } n \rightarrow \infty
$$

Since $U_{\text {ad }}$ is closed and convex, we can use Mazur's lemma to conclude that there exists a convex combination of $\left\{u_{n}\right\}$, denoted by $\left\{\bar{u}_{n}\right\}$, which strongly converges to $u^{0} \in U_{\text {ad }}$.

For every $t \in[0, b]$, we know that $x^{n}\left(t^{n}, f^{n}, u^{n}\right)$ can be rewritten as

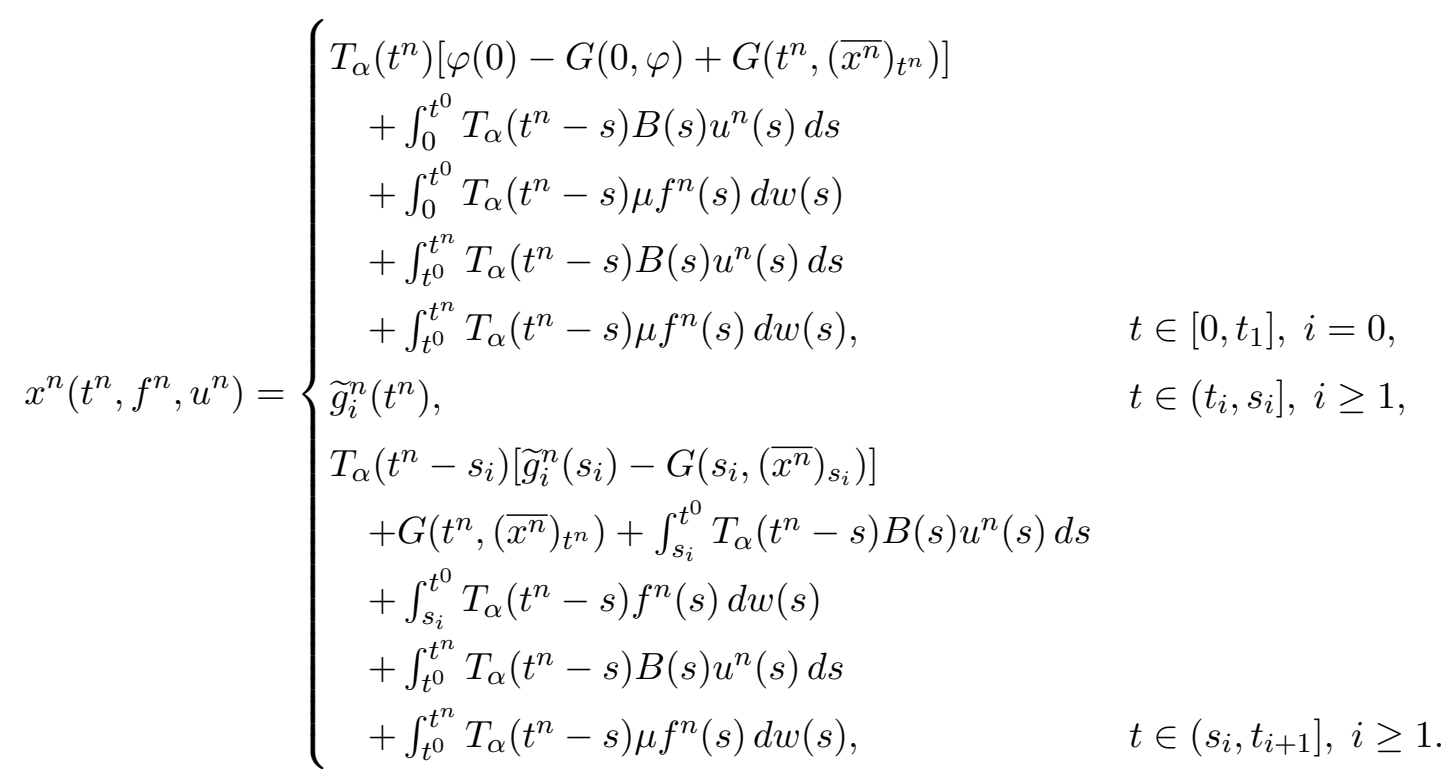

By (H1), (H2) and (H4), we have

$$
\begin{aligned}
T_{\alpha}\left(t^{n}\right)[\varphi(0)-G(0, \varphi)] & \rightarrow T_{\alpha}\left(t^{0}\right)[\varphi(0)-G(0, \varphi)] \quad \text { as } n \rightarrow \infty, \\
G\left(t^{n},\left(\overline{x^{n}}\right)_{t^{n}}\right) & \rightarrow G\left(t^{0},\left(\overline{x^{0}}\right)_{t^{0}}\right) \quad \text { as } n \rightarrow \infty, \\
\widetilde{g}_{i}^{n}\left(t^{n}\right) & \rightarrow \widetilde{g}_{i}^{0}\left(t^{0}\right) \quad \text { as } n \rightarrow \infty, \\
T_{\alpha}\left(t^{n}-s_{i}\right)\left[\widetilde{g}_{i}^{n}\left(s_{i}\right)-G\left(s_{i},\left(\overline{x^{n}}\right)_{s_{i}}\right)\right] & \rightarrow T_{\alpha}\left(t^{0}-s_{i}\right)\left[\widetilde{g}_{i}^{0}\left(s_{i}\right)-G\left(s_{i},\left(\overline{x^{0}}\right)_{s_{i}}\right)\right] \quad \text { as } n \rightarrow \infty,
\end{aligned}
$$

where $\widetilde{g}_{i}^{0}\left(t^{0}\right) \in g_{i}\left(t^{0},\left(\overline{x^{0}}\right) t^{0}\right)$. It follows from condition (H3), Lemma 2.9 and Höder's inequality that

$$
\begin{aligned}
E & \left\|\int_{t^{0}}^{t^{n}} T_{\alpha}\left(t^{n}-s\right) B(s) u^{n}(s) d s+\int_{t^{0}}^{t^{n}} T_{\alpha}\left(t^{n}-s\right) \mu f^{n}(s) d w(s)\right\|_{H}^{p} \\
\leq & 2^{p-1} M^{p}\|B\|_{\infty}^{p}\left(t^{n}-t^{0}\right)^{p-1}\|u\|_{L_{\mathcal{F}}^{p}\left(\left[0, t_{1}\right], Y\right)}^{p} \\
& +2^{p-1} C_{p} M^{p}\|\mu\|_{L(K, H)}^{p}\left(t^{n}-t^{0}\right)^{p / 2-1}\left\|\Lambda_{n^{*}}\right\|_{L^{1}\left(\left[0, t_{1}\right], \mathbb{R}^{+}\right)} .
\end{aligned}
$$


One has

$$
\begin{aligned}
& \int_{t^{0}}^{t^{n}} T_{\alpha}\left(t^{n}-s\right) B(s) u^{n}(s) d s \rightarrow 0 \quad \text { as } n \rightarrow \infty \\
& \int_{t^{0}}^{t^{n}} T_{\alpha}\left(t^{n}-s\right) \mu f^{n}(s) d w(s) \rightarrow 0 \quad \text { as } n \rightarrow \infty .
\end{aligned}
$$

By strongly continuity of $B$, we have

$$
\left\|B u^{n}-B u^{0}\right\|_{H}^{p} \rightarrow 0 \quad \text { as } n \rightarrow \infty .
$$

It follows that

$$
E\left\|\int_{s_{i}}^{t} S_{\alpha}(t-s)\left[B(s) u^{n}(s)-B(s) u^{*}(s)\right] d s\right\|_{H}^{p} \leq M^{p}\left(t_{i+1}-s_{i}\right)^{p}\left\|B u^{n}-B u^{0}\right\|_{H}^{p},
$$

which implies that

$$
\int_{s_{i}}^{t} S_{\alpha}(t-s) B(s) u^{n}(s) d s \rightarrow \int_{s_{i}}^{t} S_{\alpha}(t-s) B(s) u^{0}(s) d s \quad \text { as } n \rightarrow \infty .
$$

By condition (H3), we can deduce that $\left\{f^{n}(s)\right\} \subseteq \Psi\left(\left(\overline{x^{n}}\right)_{s}\right)$ is bounded on $L^{p}([0, b], H)$. Therefore, by the reflexivity of $L^{p}([0, b], H)$, then there exist a subsequence of $\left\{f^{n}\right\}$, denoted again by $\left\{f^{n}\right\}$, and $f^{0} \in L^{p}([0, b], H)$, such that

$$
f^{n} \stackrel{w}{\longrightarrow} f^{0} \quad \text { in } L^{p}([0, b], H) \quad \text { as } n \rightarrow \infty .
$$

By the compactness of $\mu$, we have

$$
\left\|\mu\left[f^{n}(s)-f^{0}(s)\right]\right\|_{H}^{p} \rightarrow 0 \quad \text { as } n \rightarrow \infty .
$$

It follows that

$$
E\left\|\int_{s_{i}}^{t} T_{\alpha}(t-s)\left[\mu f^{n}(s)-\mu f^{0}(s)\right] d w(s)\right\|_{H}^{p} \leq C_{p} M^{p}\left(t_{i+1}-s_{i}\right)^{p / 2}\left\|\mu\left[f^{n}(s)-f^{0}(s)\right]\right\|_{H}^{p} .
$$

Thus, from Lemma 3.1, it follows that

$$
\int_{s_{i}}^{t} T_{\alpha}(t-s) \mu f^{n}(s) d w(s) \rightarrow \int_{s_{i}}^{t} T_{\alpha}(t-s) \mu f^{0}(s) d w(s) \quad \text { as } n \rightarrow \infty
$$

where $f^{0} \in \Psi\left(\left(\overline{x^{0}}\right)_{s}\right)$. Hence,

$$
x_{1}=x\left(t^{0}, f^{0}, u^{0}\right)
$$




$$
= \begin{cases}T_{\alpha}\left(t^{0}\right)\left[\varphi(0)-G(0, \varphi)+G\left(t^{0},\left(\overline{x^{0}}\right)_{t^{0}}\right)\right] & \\ \quad+\int_{0}^{t^{n}} T_{\alpha}\left(t^{0}-s\right) B(s) u^{0}(s) d s & \\ \quad+\int_{0}^{t^{0}} T_{\alpha}\left(t^{0}-s\right) \mu f^{0}(s) d w(s), & t \in\left[0, t_{1}\right], i=0, \\ \widetilde{g}_{i}^{0}\left(t^{0}\right), & t \in\left(t_{i}, s_{i}\right], i \geq 1, \\ T_{\alpha}\left(t^{0}-s_{i}\right)\left[\widetilde{g}_{i}^{0}\left(s_{i}\right)-G\left(s_{i},\left(\overline{x^{0}}\right)_{s_{i}}\right)\right]+G\left(t^{n},\left(\overline{x^{0}}\right)_{t^{n}}\right) & \\ \quad+\int_{s_{i}}^{t^{0}} T_{\alpha}\left(t^{0}-s\right) B(s) u^{0}(s) d s & \\ \quad+\int_{s_{i}}^{t^{0}} T_{\alpha}\left(t^{0}-s\right) \mu f^{0}(s) d w(s), & t \in\left(s_{i}, t_{i+1}\right], i \geq 1,\end{cases}
$$

where $f^{0}(s) \in \Psi\left(\left(\overline{x^{0}}\right)_{s}\right), \widetilde{g}_{i}^{0}\left(t^{0}\right) \in g_{i}\left(t^{0},\left(\overline{x^{0}}\right)_{t^{0}}\right)$, that is, $u^{0}$ is the time optimal control, and $x\left(t^{0}, f^{0}, u^{0}\right)$ is just the trajectory corresponding to the control $u^{0}$ and the proof is complete.

\section{An example}

In this section, we provide an example which comes from nonconvex problems for semipermeable media and leads to a fractional stochastic differential inclusions with Clarke's subdifferential of type (1.1)-(1.3). We remark that the nonmonotone semipermeability conditions are realized by various types of membranes, natural and artificial ones. These conditions arise in electrostatics, hydraulics and in the description of the flow of Binghams fluids. Here, a practical method to describe the system with fractional neutral functional differential inclusions with non-instantaneous impulses and Clarke subdifferential aims at reality in nonconvex semipermeable system identification and control, as real systems are distributed phenomena. We study this type of system with hereditary control variable and obtained the time optimal control of the system.

Consider the following fractional non-instantaneous impulsive stochastic partial differential systems of the form

$$
\begin{aligned}
& { }^{c} D_{t}^{2 / 3}\left[z(t, x)-\int_{-\infty}^{0} e^{2 s} \frac{|z(t+s, x)|}{1+|z(t+s, x)|} d s\right] \\
& \in\left(\sum_{|\varsigma| \leq 2 m} a_{\varsigma}(x) D^{\varsigma}\right)\left[z(t, x)-\int_{-\infty}^{0} e^{2 s} \frac{|z(t+s, x)|}{1+|z(t+s, x)|} d s\right] \\
& +J_{t}^{1 / 3} \int_{\Pi} \sin (x, y) u(t, y) d y \\
& +J_{t}^{1 / 3}\left[\left(\int_{\Pi} \cos (x, y) d y\right)\left(\partial \int_{-\infty}^{0} e^{4 s} \sin (|z(t+s, x)|) d s\right)\right] \frac{d w(t)}{d t}, \\
& \quad t \in[0,1 / 2) \cup[3 / 4,1], x \in \Pi,
\end{aligned}
$$




$$
\begin{gathered}
z(t, x)=0, \quad(t, x) \in[0,1] \times \partial \Pi, \\
z(\tau, x)=\varphi(\tau, x), \quad(\tau, x) \in(-\infty, 0] \times \Pi, \\
z(t, x) \in \int_{-\infty}^{0} t^{2} e^{2 s}|z(t+s, x)| d s, \quad t \in(1 / 2,3 / 4], x \in \Pi,
\end{gathered}
$$

where ${ }^{c} D_{t}^{2 / 3}$ is a Caputo fractional partial derivative of order $\alpha=2 / 3 \in(0,1), \varphi$ is continuous, and $\Pi$ is a bounded domain in $\mathbb{R}^{n}$ with a smooth boundary $\partial \Pi, a_{\varsigma}(x)$ is a smooth real function on $\bar{\Pi}, D^{\varsigma}=D_{x_{1}}^{\varsigma} \cdots D_{x_{n}}^{\varsigma}, D_{x_{k}}=\frac{\partial}{\partial x_{k}}, \varsigma=\left(\varsigma_{1}, \ldots, \varsigma_{n}\right)$ is an $n$ dimensional multi-index, $|\varsigma|=\varsigma_{1}+\cdots+\varsigma_{n} .0=t_{0}=s_{0}, t_{1}=1 / 2, s_{1}=3 / 4, t_{2}=1$ are fixed real numbers, and $w(t)$ denotes a one-dimensional standard Wiener process.

The differential operator $L_{m}=\sum_{|\varsigma| \leq 2 m} a_{\varsigma}(x) D^{\varsigma}$ is strongly elliptic (see [32]). Let $H=Y=\{\omega: \omega \in C(\bar{\Pi}), \omega=0$ on $\partial \Pi\}$ with the norm $\|\cdot\|_{C(\bar{\Pi})}$ and $D(A)=\{\omega:$ $\omega \in D\left(A_{\infty}\right), L_{m} \omega \in C(\bar{\Pi}), L_{m} \omega=0$ on $\left.\partial \Pi\right\}$ define the operator $A: D(A) \rightarrow H$ by $A v=$ $L_{m} v$, where $D\left(A_{\infty}\right)=\left\{\omega: \omega \in W^{2 m, p}(\Pi)\right\}$ for all $p>n$. It is well known that $A$ generates an analytic, noncompact semigroup $(T(t))_{t \geq 0}$ on $H$. From the subordination principle [3, Theorems 3.1 and 3.3], we know that $A$ is the infinitesimal generator of an $\alpha$-order fractional bounded and analytic resolvent operator $T_{\alpha}(t)(t \geq 0)$ of analyticity type $\left(\omega_{0}, \theta_{0}\right)$. Therefore, the linear system corresponding to (5.1)-(5.4) has an associated solution operator $T_{\alpha}(\cdot)$ on $H$. We define the admissible control set $U_{\text {ad }}=\{u(\cdot, y) \mid \mathbb{R} \rightarrow$

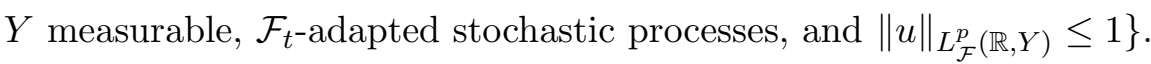

Let the phase space $\mathcal{B}$ be

$$
\mathcal{B}=C_{\bar{\gamma}}=\left\{\psi \in C((-\infty, 0], H): \lim _{\theta \rightarrow-\infty} e^{\bar{\gamma} \theta} \psi(\theta) \text { exists in } H\right\},
$$

where $\bar{\gamma}>0$ and set

$$
\|\psi\|_{\mathcal{B}}=\sup _{-\infty<\theta \leq 0} e^{\bar{\gamma} \theta}\|\psi(\theta)\|_{C(\bar{\Pi})}, \quad \psi \in C_{\bar{\gamma}}
$$

It is well known that $C_{\bar{\gamma}}$ satisfies the Axioms $(\mathrm{A})-(\mathrm{C})$ with $\widetilde{H}=1, \widetilde{K}(t)=\max \left\{1, e^{-\bar{\gamma} t}\right\}$ and $\widetilde{M}(t)=e^{-\bar{\gamma} t}$ (see $[21$, Theorem 1.3.7] for details).

Additionally, we choose $0<\bar{\gamma}<2$ and take $\psi(\theta)(x)=\psi(\theta, x),(\theta, x) \in(-\infty, 0] \times \mathcal{B}$. Let $z(s)(x)=z(s, x), G:[0,1] \times \mathcal{B} \rightarrow H, F:[0,1] \times \mathcal{B} \rightarrow \mathbb{R}, g_{i}:(1 / 2,3 / 4] \times \mathcal{B} \rightarrow \mathcal{P}(H)$ be the operators defined by

$$
\begin{array}{cc}
G(t, \psi)(x)=\int_{-\infty}^{0} e^{2 s} \frac{|\psi(s)(x)|}{1+|\psi(s)(x)|} d s, & \mathcal{N}(t, \psi)(x)=\psi(0) x-G(t, \psi)(x), \\
F(t, \psi)(x)=\int_{-\infty}^{0} e^{4 s} \sin (|\psi(s)(x)|) d s, & g_{1}(t, \psi)(x)=\int_{-\infty}^{0} t^{2} e^{2 s}|\psi(s)(x)| d s .
\end{array}
$$

For all $u \in C([0,1] \times \bar{\Pi})$, we define an operator $B$ as follows:

$$
(B u)(t)(x)=\int_{\Pi} \sin (x, y) u(t, y) d y, \quad \mu(x)=\int_{\Pi} \cos (x, y) d y .
$$


Using these definitions, we can represent the system $(5.1)-(5.4)$ in the abstract form 1.1 (1.3). Moreover, we have for all $(t, \psi),\left(t_{1}, \psi_{1}\right) \in[0,1] \times \mathcal{B}$,

$$
\begin{aligned}
E\left\|G(t, \psi)-G\left(t_{1}, \psi_{1}\right)\right\|_{C(\bar{\Pi})}^{p} & =E\left[\sup _{x \in \bar{\Pi}} \int_{-\infty}^{0} e^{2 s}\left(\frac{|\psi(s)(x)|}{1+|\psi(s)(x)|}-\frac{\left|\psi_{1}(s)(x)\right|}{1+\left|\psi_{1}(s)(x)\right|}\right) d s\right]^{p} \\
& \leq E\left[\int_{-\infty}^{0} e^{(2-\bar{\gamma}) s} e^{\bar{\gamma} s}\left\|\psi(s)-\psi_{1}(s)\right\|_{C(\bar{\Pi})} d s\right]^{p} \\
& \leq\left(\int_{-\infty}^{0} e^{(2-\bar{\gamma}) s} d s\right)^{p}\left\|\psi-\psi_{1}\right\|_{\mathcal{B}}^{p} \\
& \leq \widetilde{L}_{G}\left\|\psi-\psi_{1}\right\|_{\mathcal{B}}^{p},
\end{aligned}
$$

and $E\|h(t, \psi)\|^{p} \leq \widetilde{L}_{G}\|\psi\|_{\mathcal{B}}^{p}$, where $\widetilde{L}_{G}=\frac{1}{(2-\bar{\gamma})^{p}}$. For all $(t, \psi),\left(t, \psi_{1}\right) \in \mathbb{R} \times \mathcal{B}$, we have

$$
\begin{aligned}
\left|F(t, \psi)(x)-F\left(t, \psi_{1}\right)(x)\right| & \leq \int_{-\infty}^{0} e^{4 s}\left|\sin (|\psi(s)(x)|)-\sin \left(\left|\psi_{1}(s)(x)\right|\right)\right| d s \\
& \leq \int_{-\infty}^{0} e^{4 s}\left|\psi(s)(x)-\psi_{1}(s)(x)\right| d s \\
& \leq \int_{-\infty}^{0} e^{(4-\bar{\gamma}) s} e^{\bar{\gamma} s}\left\|\psi(s)-\psi_{1}(s)\right\|_{C(\bar{\Pi})} d s \\
& \leq \frac{1}{4-\bar{\gamma}}\left\|\psi-\psi_{1}\right\|_{\mathcal{B} .}
\end{aligned}
$$

For any $\lambda>0$, and $\psi, \psi_{1} \in \mathcal{B}, t \in \mathbb{R}$, we have

$$
\begin{aligned}
& \left|\frac{F\left(t, \psi_{1}+\lambda \psi\right)(x)-F\left(t, \psi_{1}\right)(x)}{\lambda}\right| \\
= & \left|\frac{\int_{-\infty}^{0} e^{4 s}\left[\sin \left(\left|\psi_{1}(s)(x)+\lambda \psi(s)(x)\right|\right)-\sin \left(\left|\psi_{1}(s)(x)\right|\right)\right] d s}{\lambda}\right| \\
\leq & \left|\int_{-\infty}^{0} e^{4 s}\right| \psi(s)(x)|d s| .
\end{aligned}
$$

Let $F^{0}(t, \psi)(x)=\int_{-\infty}^{0} e^{4 s}|\psi(s)(x)| d s$. Then it is easy to verify that

$$
\begin{aligned}
E\|\partial F(t, \psi)\|_{C(\bar{\Pi})}^{p} & =E\left[\sup _{x \in \bar{\Pi}} \int_{-\infty}^{0} e^{4 s}|\psi(s)(x)| d s\right]^{p} \leq E\left[\int_{-\infty}^{0} e^{(4-\bar{\gamma}) s} e^{\widetilde{\gamma} s}\|\psi(s)\|_{C(\bar{\Pi})} d s\right]^{p} \\
& \leq\left(\int_{-\infty}^{0} e^{(4-\bar{\gamma}) s} d s\right)^{p}\|\psi\|_{\mathcal{B}}^{p} \leq \widetilde{L}_{F}\|\psi\|_{\mathcal{B}}^{p}
\end{aligned}
$$

for all $(t, \psi) \in[0,1] \times \mathcal{B}$, where $\widetilde{L}_{F}=\frac{1}{(4-\bar{\gamma})^{p}}$. For all $(t, \psi),\left(t_{1}, \psi_{1}\right) \in(1 / 2,3 / 4] \times \mathcal{B}$, we 
have

$$
\begin{aligned}
& E\left\|g_{1}(t, \psi)-g_{1}\left(t_{1}, \psi_{1}\right)\right\|_{\bar{\Pi}}^{p} \\
= & E\left[\sup _{x \in \bar{\Pi}}\left(\int_{-\infty}^{0} t^{2} e^{2 s}|\psi(s)(x)| d s-\int_{-\infty}^{0} t_{1}^{2} e^{2 s}\left|\psi_{1}(s)(x)\right| d s\right)\right]^{p} \\
\leq & E\left[\left|t^{2}-t_{1}^{2}\right| \int_{-\infty}^{0} e^{\bar{\gamma} s} d s+\left|t_{1}\right|^{2} \int_{-\infty}^{0} e^{(2-\bar{\gamma}) s} e^{\bar{\gamma} s}\left\|\psi(s)-\psi_{1}(s)\right\|_{C(\bar{\Pi})} d s\right]^{p} \\
\leq & 2^{p-1}\left[\left|t^{2}-t_{1}^{2}\right|^{p}\left(\int_{-\infty}^{0} e^{\bar{\gamma} s} d s\right)^{p}+\left|t_{1}\right|^{2 p}\left(\int_{-\infty}^{0} e^{(2-\bar{\gamma}) s} d s\right)^{p}\left\|\psi-\psi_{1}\right\|_{\mathcal{B}}^{p}\right] \\
\leq & \widetilde{\gamma}_{1}\left[\left|t-t_{1}\right|+\left\|\psi-\psi_{1}\right\|_{\mathcal{B}}^{p}\right],
\end{aligned}
$$

and $\left\|g_{1}(t, \psi)\right\|_{\bar{\Pi}}^{p} \leq \widetilde{\gamma}_{1}\|\psi\|_{\mathcal{B}}^{p}$, where $\widetilde{\gamma}_{1}=2^{p-1}\left[\left(\frac{3}{2 \bar{\gamma}}\right)^{p}+\left(\frac{3}{4}\right)^{2 p} \frac{1}{(2-\bar{\gamma})^{p}}\right]$. Similarly, we have $\|\mu\|_{C(\bar{\Pi})}^{p} \leq\left(\sup _{x \in \bar{\Pi}} \int_{\bar{\Pi}} \cos (x, y) d y\right)^{p}$. Therefore, Assumptions (H1)-(H4) all hold in Section 3. On the other hand, for $u \in C([0,1] \times \bar{\Pi})$, we have

$$
\|B u\|_{C([0,1] \times \bar{\Pi})}^{p}=\left[\sup _{x \in \bar{\Pi}}\left(\int_{\bar{\Pi}} \sin (x, y) u(t, y) d y\right)\right]^{p} \leq \widetilde{L}_{u}\|u\|_{C([0,1] \times \bar{\Pi})}^{p},
$$

where $\widetilde{L}_{u}=\left(\sup _{x \in \bar{\Pi}} \int_{\bar{\Pi}} \sin (x, y) d y\right)^{p}$. Then, we can conclude that $B \in L^{\infty}([0,1], L(H))$. Let's take $K_{b}=M_{b}=b=1, L_{G}=\widetilde{L}_{G}, \gamma_{i}=\widetilde{\gamma}_{1}, i=1, \ldots, N$. It is easy to see that

$$
2^{p-1}\left[\left(4^{p-1} M^{p}+1\right) \widetilde{\gamma}_{1}+2^{p-1}\left(1+M^{p}\right) \widetilde{L}_{G}\right]<1,
$$

whenever $\widetilde{L}_{G}$ and $\widetilde{\gamma}_{1}$ are small enough. Now the condition (3.1) holds. Further, all the conditions in Theorem 4.1 satisfied. Hence by Theorem 4.1, the system (5.1)-(5.4) has at least one time optimal pair.

\section{References}

[1] N. U. Ahmed, Optimal control of impulsive stochastic evolution inclusions, Discuss. Math. Differ. Incl. Control Optim. 22 (2002), no. 2, 155-184.

[2] L. Bai, J. J. Nieto and J. M. Uzal, On a delayed epidemic model with noninstantaneous impulses, Commun. Pure Appl. Anal. 19 (2020), no. 4, 1915-1930.

[3] E. G. Bajlekova, Fractional Evolution Equations in Banach Spaces, Ph.D. Dissertation, Eindhoven University of Technology, Eindhoven, 2001.

[4] P. Balasubramaniam and P. Tamilalagan, The solvability and optimal controls for impulsive fractional stochastic integro-differential equations via resolvent operators, J. Optim. Theory Appl. 174 (2017), no. 1, 139-155. 
[5] J. E. Brandeberry, Infinite time optimal control for a class of stochastic systems, Internat. J. Systems Sci. 4 (1973), 417-427.

[6] S. Carl and D. Motreanu, Extremal solutions of quasilinear parabolic inclusions with generalized Clarke's gradient, J. Differential Equations 191 (2003), no. 1, 206-233.

[7] F. H. Clarke, Optimization and Nonsmooth Analysis, Canadian Mathematical Society Series of Monographs and Advanced Texts, A Wiley-Interscience Publication, John Wiley \& Sons, New York, 1983.

[8] G. Da Prato and J. Zabczyk, Stochastic Equations in Infinite Dimensions, Encyclopedia of Mathematics and its Applications 44, Cambridge University Press, Cambridge, 1992.

[9] A. Debbouche and J. J. Nieto, Sobolev type fractional abstract evolution equations with nonlocal conditions and optimal multi-controls, Appl. Math. Comput. 245 (2014), 7485 .

[10] A. Debbouche, J. J. Nieto and D. F. M. Torres, Optimal solutions to relaxation in multiple control problems of Sobolev type with nonlocal nonlinear fractional differential equations, J. Optim. Theory Appl. 174 (2017), no. 1, 7-31.

[11] A. Debbouche and D. F. M. Torres, Sobolev type fractional dynamic equations and optimal multi-integral controls with fractional nonlocal conditions, Fract. Calc. Appl. Anal. 18 (2015), no. 1, 95-121.

[12] K. Deimling, Multivalued Differential Equations, De Gruyter Series in Nonlinear Analysis and Applications 1, Walter de Gruyter, Berlin, 1992.

[13] R. Dhayal, M. Malik, S. Abbas and A. Debbouche, Optimal controls for secondorder stochastic differential equations driven by mixed-fractional Brownian motion with impulses, Math. Methods Appl. Sci. 43 (2020), no. 7, 4107-4124.

[14] H. O. Fattorini, Time-optimal control of solutions of operational differential equations, J. SIAM Control Ser. A 2 (1964), 54-59.

[15] L. Górniewicz, Topological Fixed Point Theory of Multivalued Mappings, Second edition, Topological Point Theory and Its Applications 4, Springer, Dordrecht, 2006.

[16] S. Guillaume, Subdifferential evolution inclusion in nonconvex analysis, Positivity 4 (2000), no. 4, 357-395.

[17] J. K. Hale and J. Kato, Phase space for retarded equations with infinite delay, Funkcial. Ekvac. 21 (1978), no. 1, 11-41. 
[18] A. Harrat, J. J. Nieto and A. Debbouche, Solvability and optimal controls of impulsive Hilfer fractional delay evolution inclusions with Clarke subdifferential, J. Comput. Appl. Math. 344 (2018), 725-737.

[19] E. Hernández and D. O'Regan, On a new class of abstract impulsive differential equations, Proc. Amer. Math. Soc. 141 (2013), no. 5, 1641-1649.

[20] E. Hernández, M. Pierri and D. O'Regan, On abstract differential equations with non instantaneous impulses, Topol. Methods Nonlinear Anal. 46 (2015), no. 2, 1067-1088.

[21] Y. Hino, S. Murakami and T. Naito, Functional-differential Equations with Infinite Delay, Lecture Notes in Mathematics 1473, Springer-Verlag, Berlin, 1991.

[22] J.-M. Jeong and S.-J. Son, Time optimal control of semilinear control systems involving time delays, J. Optim. Theory Appl. 165 (2015), no. 3, 793-811.

[23] Y.-r. Jiang and N.-j. Huang, Solvability and optimal controls of fractional delay evolution inclusions with Clarke subdifferential, Math. Methods Appl. Sci. 40 (2017), no. 8, 3026-3039.

[24] S. Kumar, Mild solution and fractional optimal control of semilinear system with fixed delay, J. Optim. Theory Appl. 174 (2017), no. 1, 108-121.

[25] V. Lakshmikantham, D. D. Baŭnov and P. S. Simeonov, Theory of Impulsive Differential Equations, Series in Modern Applied Mathematics 6, World Scientitic, Singapore, 1989.

[26] D. Li, F. Chen, Y. Wu and Y. An, Multiple solutions for a class of p-Laplacian type fractional boundary value problems with instantaneous and non-instantaneous impulses, Appl. Math. Lett. 106 (2020), 106352, 8 pp.

[27] T. Lian, Z. Fan and G. Li, Time optimal controls for fractional differential systems with Riemann-Liouville derivatives, Fract. Calc. Appl. Anal. 21 (2018), no. 6, 15241541.

[28] S. Liu and J. Wang, Optimal controls of systems governed by semilinear fractional differential equations with not instantaneous impulses, J. Optim. Theory Appl. 174 (2017), no. 2, 455-473.

[29] I. Matychyn and V. Onyshchenko, On time-optimal control of fractional-order systems, J. Comput. Appl. Math. 339 (2018), 245-257.

[30] S. Migórski and A. Ochal, Quasi-static hemivariational inequality via vanishing acceleration approach, SIAM J. Math. Anal. 41 (2009), no. 4, 1415-1435. 
[31] M. Muslim and A. Kumar, Controllability of fractional differential equation of order $\alpha \in(1,2]$ with non-instantaneous impulses, Asian J. Control 20 (2018), no. 2, 935942.

[32] A. Pazy, Semigroups of Linear Operators and Applications to Partial Differential Equations, Applied Mathematical Sciences 44, Springer-Verlag, New York, 1983.

[33] L. Peng, Y. Zhou and A. Debbouche, Approximation techniques of optimal control problems for fractional dynamic systems in separable Hilbert spaces, Chaos Solitons Fractals 118 (2019), 234-241.

[34] I. Podlubny, Fractional Differential Equations: An introduction to fractional derivatives, fractional differential equations, to methods of their solution and some of their applications, Mathematics in Sciences and Engineering 198, Academic Press, San Diego, CA, 1999.

[35] H. Proppe and A. Boyarsky, A time-optimal stochastic control problem, Internat. J. Systems Sci. 8 (1977), no. 10, 1193-1199.

[36] J. Prüss, Evolutionary Integral Equations and Applications, Monographs in Mathematics 87, Birkhaüser Verlag, Basel, 1993.

[37] J. Wang, M. Fečkan and A. Debbouche, Time optimal control of a system governed by non-instantaneous impulsive differential equations, J. Optim. Theory Appl. 182 (2019), no. 2, 573-587.

[38] J. Wang, X. Xiang and W. Wei, The constructive approach on existence of time optimal controls of system governed by nonlinear equations on Banach spaces, Electron. J. Qual. Theory Differ. Equ. 2009 (2009), no. 45, 10 pp.

[39] Z. Yan and X. Jia, Optimal controls of fractional impulsive partial neutral stochastic integro-differential systems with infinite delay in Hilbert spaces, Int. J. Control Automat. Syst. 15 (2017), no. 3, 1051-1068.

[40] Z. Yan and F. Lu, The optimal control of a new class of impulsive stochastic neutral evolution integro-differential equations with infinite delay, Internat. J. Control 89 (2016), no. 8, 1592-1612.

[41] _ Solvability and optimal controls of a fractional impulsive stochastic partial integro-differential equation with state-dependent delay, Acta Appl. Math. 155 (2018), 57-84. 
[42] Z. Yu, The stochastic maximum principle for optimal control problems of delay systems involving continuous and impulse controls, Automatica J. IFAC 48 (2012), no. $10,2420-2432$.

Zuomao Yan

Department of Mathematics, Hexi University, Zhangye, Gansu 734000, China

E-mail address: yanzuomao@163.com 